CRYSTALLOGRAPHIC COMMUNICATIONS

ISSN 2056-9890

Received 13 August 2018

Accepted 20 September 2018

Edited by D. Chopra, Indian Institute of Science Education and Research Bhopal, India

Keywords: octyloxy pillararene; hydroxy functionalization; crystal structure.

CCDC reference: 1868738

Supporting information: this article has supporting information at journals.iucr.org/e

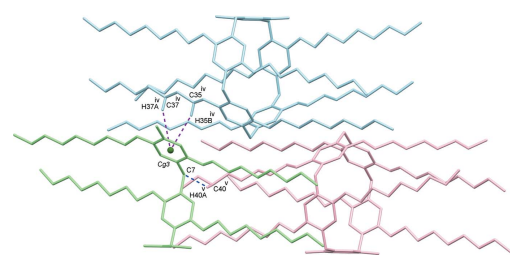

OPEN $\odot$ ACCESS

\section{Encapsulated dichloroethane-mediated interlocked supramolecular polymeric assembly of A1/A2-dihydroxy-octyloxy pillar[5]arene 1,2-dichloroethane monosolvate}

Talal F. Al-Azemi,* Mickey Vinodh, Abdirahman A. Mohamod and Fatemeh H. Alipour

Department of Chemistry, Kuwait University, PO Box 5969, Safat 13060, Kuwait. *Correspondence e-mail: t.alazemi@ku.edu.kw

Crystals of 1-(1,4-dihydroxy)-2,3,4,5-(1,4-dioctyloxy)-pillar[5]arene, $\mathrm{C}_{99} \mathrm{H}_{158} \mathrm{O}_{10} \cdot \mathrm{C}_{2} \mathrm{H}_{4} \mathrm{Cl}_{2}$, were grown from a 1,2-dicholoroethane/n-hexane solvent system. In the crystal, the encapsulated 1,2-dichloroethane solvent is stabilized by $\mathrm{C}-\mathrm{H} \cdots \pi$ interactions and mediates the formation of an interlocked supramolecular polymer via $\mathrm{C}-\mathrm{H} \cdots \mathrm{Cl}$ interactions.

\section{Chemical context}

Supramolecular polymers constructed by reversible noncovalent interactions such as hydrogen bonds, metal-ligand interactions, host-guest interactions, $\pi-\pi$ interactions and van der Waals forces have gained considerable interest for their intriguing properties of recycling and responsiveness to external stimuli (Raghupathi et al., 2014; Takashima et al., 2017). Pillararenes are unique three-dimensional macrocyclic compounds which possess symmetric rigid structures and are easy to functionalize with various substituents (Ogoshi et al., 2008; Al-Azemi et al., 2017). They exhibit outstanding abilities to selectively bind different kinds of guest molecules and thus are excellent host molecules for guest encapsulation and molecular recognition. Their unique structural features also enable them to exhibit interesting self-assembling characteristics, which make them potential candidates for use in fabricating functional materials in supramolecular systems and nanotechnology. The construction of pillararene-based supramolecular assemblies is very interesting because it raises the possibility of using these macrocycles for many important functional materials, which include enzyme models, fieldeffect transistors, gas sensors or photovoltaic cells (Han et al., 2015; Pan \& Xue, 2013; Hu et al., 2016; Zhang et al., 2018).

Supramolecular motifs such as hydrogen bonding or hostguest interactions can be employed to promote the selfassembly of pillararene analogues. The introduction of appropriate peripheral functionalization at the macrocycle will give rise to numerous features that also allow their organization at a supramolecular level (Xue et al., 2012). The characteristics of the encapsulated guest molecules can also be utilized to tune the supramolecular nature of these macromolecules. The present work discusses the crystal structure of a pillararene system, Pil-OctOH. $\mathbf{C}_{2} \mathbf{H}_{\mathbf{4}} \mathbf{C l}_{2}$, which possesses two hydroxy groups at the macrocyclic periphery. The remaining apical sites on the pillararene are functionalized with long 
$n$-octyloxy substituents. The role of the guest molecule in the formation of an interlocked supramolecular polymer via various supramolecular interactions is also described.

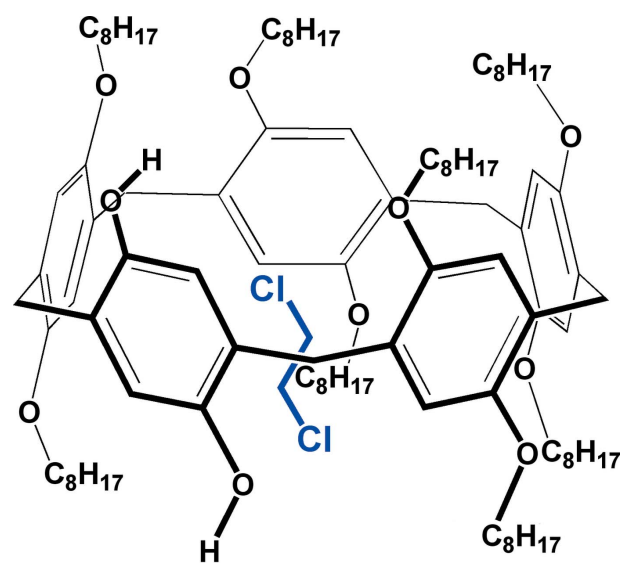

\section{Structural commentary}

Fig. 1 shows the structure of the title A1/A2-dihydroxy-octyloxy-pillar[5]arene (Pil-OctOH). The asymmetric unit contains half of the molecule and the whole structure is generated by twofold rotation symmetry (symmetry operation: $\left.-x+1, y,-z+\frac{1}{2}\right)$. The 1,2 dichloroethane solvent is encapsulated within the pillararene cavity. The basic pillar[5]arene macrocycle is a pentagon with an average corner-to-centroid distance of $4.99 \AA$. As a result of the presence of eight linear $n$-octyloxy chains at its apical positions, this novel pillararene could be considered to be a long cylindrical-shaped functional molecule where the long tail
Table 1

Hydrogen-bond geometry $\left(\AA{ }^{\circ}\right)$.

\begin{tabular}{lllll}
\hline$D-\mathrm{H} \cdots A$ & $D-\mathrm{H}$ & $\mathrm{H} \cdots A$ & $D \cdots A$ & $D-\mathrm{H} \cdots A$ \\
\hline O5-H5 $\cdots \mathrm{O} 1$ & $0.85(2)$ & $1.93(2)$ & $2.754(2)$ & $165(2)$ \\
C34 ${ }^{\mathrm{i}}-\mathrm{H} 34 B^{\mathrm{i}} \cdots \mathrm{Cl} 1$ & 0.98 & 2.90 & $3.782(3)$ & 151 \\
\hline
\end{tabular}

Symmetry code: (i) $-x+\frac{3}{2},-y+\frac{3}{2},-z$.

ends are hydrophobic in nature. Additionally, the presence of hydroxy groups at two apical positions provides a hydrophilic pocket in the vicinity of the pillararene macrocycle. The hydroxyl groups are observed to be engaged in intramolecular hydrogen bonds with the oxygen atoms of the adjacent octyloxy moieties via $\mathrm{O}-\mathrm{H} \cdots \mathrm{O}$ interactions (Fig. 1 and Table 1).

\section{Supramolecular features}

In the title macrocyclic compound, the encapsulated 1,2-dichloroethane solvent is stabilized inside the cavity by $\mathrm{C}-$ $\mathrm{H} \cdots \pi$ interactions with the pillararene aromatic ring (Table 2). Interestingly, the guest 1,2-dichloroethane facilitates the formation of a supramolecular interlocked network through efficient $\mathrm{C}-\mathrm{H} \cdots \mathrm{Cl}$ interactions (Fig. 2 and Table 1), which form chains along the $b$-axis direction. Additional stabilization of these chains is attained by dimer formation via weak $\mathrm{C}-$ $\mathrm{H}$.. C interactions between pillararene octyl chains (Fig. 2 and Table 2). Although the A1/A2 dihydroxy groups on the pillararene rim play no part in the formation of the supramolecular assembly, their small size provides an opening which enables access to the encapsulated guest molecule. The pillararene molecule in each chain interacts with neighboring

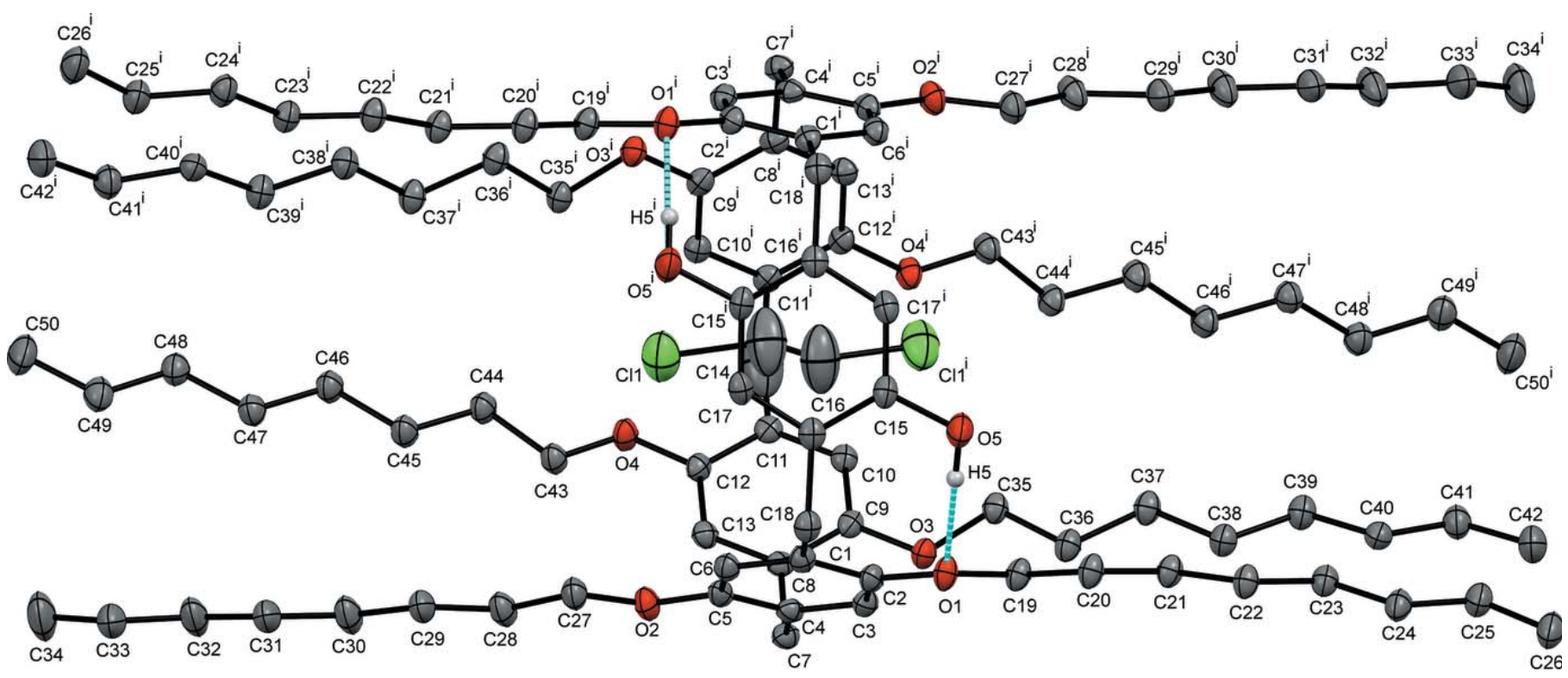

Figure 1

Displacement ellipsoid representation (30\% probability) of Pil-OctOH. $\mathbf{C}_{\mathbf{2}} \mathbf{H}_{\mathbf{4}} \mathbf{C l}_{\mathbf{2}}$. Hydrogen atoms are omitted for clarity except for those of the hydroxy groups. Blue dotted lines indicate intramolecular hydrogen bonds between the hydroxy groups and the oxygen atoms of adjacent octyloxy moieties. [Symmetry code: (i) $-x+1, y,-z+\frac{1}{2}$.] 
Table 2

Summary of weak interactions $\left(\AA,^{\circ}\right)$.

$C g 1-C g 4$ are the centroids of the $\mathrm{C} 15-\mathrm{C} 17 / \mathrm{C} 15^{\mathrm{i}}-\mathrm{C} 17^{\mathrm{i}}, \mathrm{C} 1-\mathrm{C} 6, \mathrm{C} 8-\mathrm{C} 13$ and $\mathrm{C}^{\mathrm{i}}-\mathrm{C} 6^{\mathrm{i}}$ rings, respectively.

\begin{tabular}{llll}
\hline$D-\mathrm{H} \cdots A$ & $\mathrm{H} \cdots A$ & $D \cdots A$ & $D-\mathrm{H} \cdots A$ \\
\hline $\mathrm{C} 51 A-\mathrm{H} 51 B \cdots C g 1$ & 2.77 & $3.700(8)$ & 156 \\
$\mathrm{C} 51 A-\mathrm{H} 51 A \cdots C g 2$ & 3.04 & $3.850(9)$ & 140 \\
$\mathrm{C} 51 B-\mathrm{H} 51 D \cdots C g 3$ & 2.71 & $3.565(7)$ & 144 \\
$\mathrm{C} 51 B-\mathrm{H} 51 C \cdots C g 4$ & 3.11 & $4.086(7)$ & 169 \\
$\mathrm{C} 29^{\mathrm{ii}}-\mathrm{H} 29 B^{\mathrm{ii}} \cdots \mathrm{C} 33$ & 3.18 & $4.136(3)$ & 163 \\
$\mathrm{C} 35^{\mathrm{iv}}-\mathrm{H} 35 B^{\mathrm{iv}} \cdots C g 3$ & 3.13 & $4.080(2)$ & 161 \\
$\mathrm{C} 37^{\mathrm{iv}}-\mathrm{H} 37 A^{\mathrm{iv}} \cdots C g 3$ & 3.36 & $4.260(2)$ & 153 \\
$\mathrm{C} 40^{\mathrm{v}}-\mathrm{H} 40 A^{\mathrm{v}} \cdots \mathrm{C} 7$ & 2.85 & $3.686(2)$ & 143
\end{tabular}

Symmetry codes: (i) $-x+1, y,-z+\frac{1}{2}$; (ii) $-x+\frac{3}{2},-y+\frac{3}{2},-z$; (iv) $-x+1,-y+2,-z+1$; (v) $-x+1, y,-z+\frac{3}{2}$. pillararenes of adjacent chains by $\mathrm{C}-\mathrm{H} \cdots \mathrm{C}$ and $\mathrm{C}-\mathrm{H} \cdots \pi$ interactions, as given in Fig. 3 and Table 2.

\section{Synthesis and crystallization}

The synthesis of 1-(1,4-dihydroxy)-2,3,4,5 (1,4-dioctyloxy)pillar[5]arene (Pil-OctOH) has been reported earlier (AlAzemi et al., 2018). Good quality single crystals of this compound were obtained by dissolving the pillararene $(25 \mathrm{mg})$ in 1,2-dichloroethane $(0.5 \mathrm{~mL})$ in a small vial and allowing solvent diffusion by keeping this solution in a larger vial containing $n$-hexane $(5 \mathrm{ml})$. Within three days, crystals of

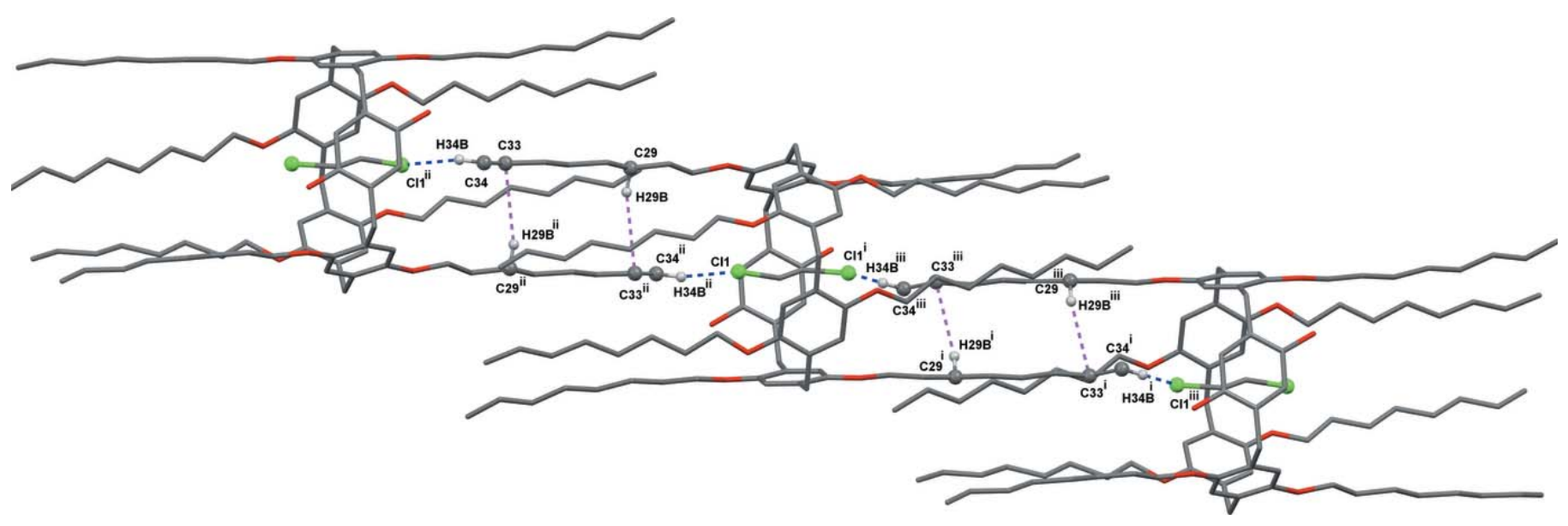

Figure 2

Supramolecular propagation of Pil-OctOH moieties as one-dimensional chains mediated by dichloroethane molecules via $\mathrm{C}-\mathrm{H} \cdots \mathrm{Cl}$ and $\mathrm{C}-\mathrm{H} \cdots \mathrm{C}$ interactions. $\mathrm{C}-\mathrm{H} \cdots \mathrm{Cl}$ interactions are represented in blue and $\mathrm{C}-\mathrm{H} \cdots \mathrm{C}$ interactions in purple. [Symmetry codes: (i) $-x+1, y,-z+\frac{1}{2}$; (ii) $-x+\frac{3}{2}$, $-y+\frac{3}{2},-z ;$ (iii) $\left.x-\frac{1}{2},-y+\frac{3}{2}, z+\frac{1}{2}\right]$

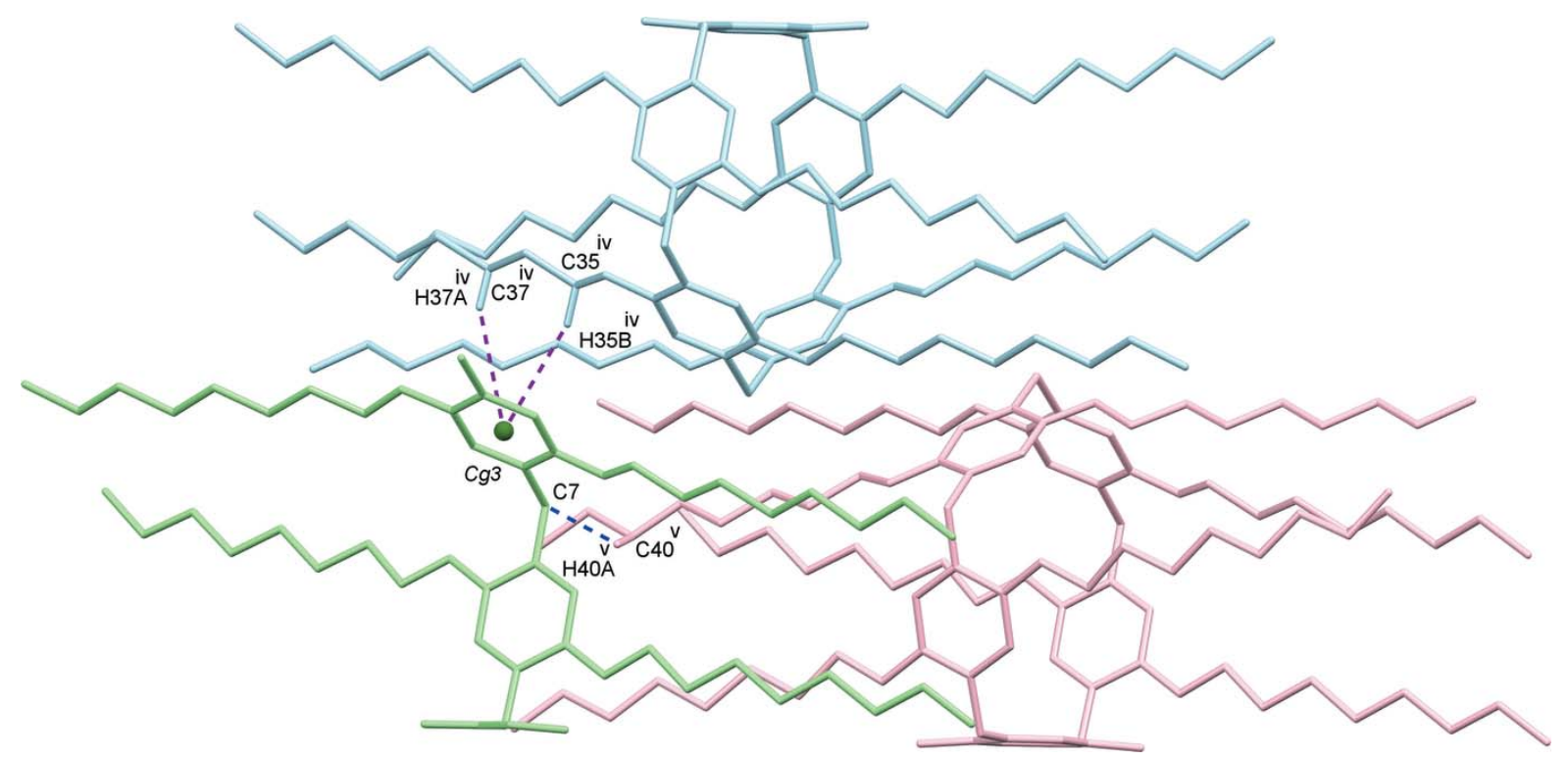

Figure 3

Adjacent pillararene fragments are connected by weak $\mathrm{C}-\mathrm{H} \cdots \mathrm{C}$ and $\mathrm{C}-\mathrm{H} \cdots \pi$ interactions in the crystal. Those interactions that are involved in supramolecular pillararene chain formation are omitted for clarity. $C g 3$ is the centroid of the $\mathrm{C} 8-\mathrm{C} 13$ ring. [Symmetry codes: (iv) $-x+1,-y+2,-z+1$; (v) $-x+1, y,-z+\frac{3}{2}$.] 
the title compound of a suitable size for diffraction analysis had formed.

\section{Refinement}

Crystal data, data collection and structure refinement details are summarized in Table 3. The $\mathrm{OH}$ hydrogen atoms were located in the electron density map. All other hydrogen atoms were placed at calculated positions and refined using a riding model with $\mathrm{C}-\mathrm{H}=0.95-0.99 \AA$ and $U_{\text {iso }}(\mathrm{H})=1.2$ or $1.5 U_{\text {eq }}(\mathrm{C})$.

\section{Funding information}

The support of the Kuwait University (research grant No. SC 03/16) and the facilities of RSPU through grant Nos. GS 03/08 (Rigaku RAPID II, Japan), GS 01/03 (NMR-Bruker DPX Avance 600, Germany and GC MS Thermo Scientific, Germany) are gratefully acknowledged.

\section{References}

Al-Azemi, T. F., Mohamod, A. A., Vinodh, M. \& Alipour, F. H. (2018). Org. Chem. Front. 5, 10-18.

Al-Azemi, T. F., Vinodh, M., Alipour, F. H. \& Mohamod, A. A. (2017). J. Org. Chem. 82, 10945-10952.

Burla, M. C., Caliandro, R., Camalli, M., Carrozzini, B., Cascarano, G. L., De Caro, L., Giacovazzo, C., Polidori, G., Siliqi, D. \& Spagna, R. (2007). J. Appl. Cryst. 40, 609-613.

Han, J., Hou, X., Ke, C., Zhang, H., Strutt, N. L., Stern, C. L. \& Stoddart, J. F. (2015). Org. Lett. 17, 3260-3263.

Higashi, T. (1995). ABSCOR. Rigaku Corporation, Tokyo, Japan.

Hu, W. B., Hu, W. J., Zhao, X. L., Liu, Y. A., Li, J. S., Jiang, B. \& Wen, K. (2016). J. Org. Chem. 81, 3877-3881.

Hübschle, C. B., Sheldrick, G. M. \& Dittrich, B. (2011). J. Appl. Cryst. 44, 1281-1284.

Macrae, C. F., Edgington, P. R., McCabe, P., Pidcock, E., Shields, G. P., Taylor, R., Towler, M. \& van de Streek, J. (2006). J. Appl. Cryst. 39, 453-457.

Ogoshi, T., Kanai, S., Fujinami, S., Yamagishi, T. A. \& Nakamoto, Y. (2008). J. Am. Chem. Soc. 130, 5022-5023.

Pan, M. \& Xue, M. (2013). Eur. J. Org. Chem. pp. 4787-4793.

Raghupathi, K. R., Guo, J., Munkhbat, O., Rangadurai, P. \& Thayumanavan, S. (2014). Acc. Chem. Res. 47, 2200-2211.
Table 3

Experimental details.

\begin{tabular}{|c|c|}
\hline \multicolumn{2}{|l|}{ Crystal data } \\
\hline Chemical formula & $\mathrm{C}_{99} \mathrm{H}_{158} \mathrm{O}_{10} \cdot \mathrm{C}_{2} \mathrm{H}_{4} \mathrm{Cl}_{2}$ \\
\hline$M_{\mathrm{r}}$ & 1607.20 \\
\hline Crystal system, space group & Monoclinic, $C 2 / c$ \\
\hline Temperature (K) & 150 \\
\hline$a, b, c(\AA)$ & $\begin{array}{l}31.4629(12), 20.2692(7) \\
\quad 15.3703(11)\end{array}$ \\
\hline$\beta\left({ }^{\circ}\right)$ & $91.275(6)$ \\
\hline$V\left(\AA^{6}\right)^{3}$ & $9799.7(9)$ \\
\hline$Z$ & 4 \\
\hline Radiation type & Мo $K \alpha$ \\
\hline$\mu\left(\mathrm{mm}^{-1}\right)$ & 0.12 \\
\hline Crystal size $(\mathrm{mm})$ & $0.21 \times 0.13 \times 0.09$ \\
\hline \multicolumn{2}{|l|}{ Data collection } \\
\hline Diffractometer & Rigaku R-AXIS RAPID \\
\hline Absorption correction & $\begin{array}{l}\text { Multi-scan (ABSCOR; Higashi, } \\
\text { 1995) }\end{array}$ \\
\hline$T_{\min }, T_{\max }$ & $0.774,0.989$ \\
\hline $\begin{array}{l}\text { No. of measured, independent and } \\
\text { observed }[I>2 \sigma(I)] \text { reflections }\end{array}$ & $42520,9959,7023$ \\
\hline$R_{\text {int }}$ & 0.032 \\
\hline$(\sin \theta / \lambda)_{\max }\left(\AA^{-1}\right)$ & 0.624 \\
\hline \multicolumn{2}{|l|}{ Refinement } \\
\hline$R\left[F^{2}>2 \sigma\left(F^{2}\right)\right], w R\left(F^{2}\right), S$ & $0.049,0.137,1.08$ \\
\hline No. of reflections & 9959 \\
\hline No. of parameters & 528 \\
\hline No. of restraints & 24 \\
\hline $\mathrm{H}$-atom treatment & $\begin{array}{l}\mathrm{H} \text { atoms treated by a mixture of } \\
\text { independent and constrained } \\
\text { refinement }\end{array}$ \\
\hline$\Delta \rho_{\max }, \Delta \rho_{\min }\left(\mathrm{e} \AA^{-3}\right)$ & $0.39,-0.43$ \\
\hline
\end{tabular}

Computer programs: CrystalClear-SM Expert (Rigaku, 2009), CrystalStructure (Rigaku, 2010), Il Milione (Burla et al., 2007), SHELXL2017/1 (Sheldrick, 2015), ShelXle (Hübschle et al., 2011) and Mercury (Macrae et al., 2006).

Rigaku (2009). CrystalClear-SM Expert. Rigaku Corporation, Tokyo, Japan.

Rigaku (2010). CrystalStructure. Rigaku Corporation, Tokyo, Japan. Sheldrick, G. M. (2015). Acta Cryst. A71, 3-8.

Takashima, Y., Yonekura, K., Koyanagi, K., Iwaso, K., Nakahata, M., Yamaguchi, H. \& Harada, A. (2017). Macromolecules, 50, 41444150.

Xue, M., Yang, Y., Chi, X., Zhang, Z. \& Huang, F. (2012). Acc. Chem. Res. 45, 1294-1308.

Zhang, H., Liu, Z. \& Zhao, Y. (2018). Chem. Soc. Rev. 47, 5491-5528. 


\section{supporting information}

Acta Cryst. (2018). E74, 1471-1474 [https://doi.org/10.1107/S2056989018013415]

Encapsulated dichloroethane-mediated interlocked supramolecular polymeric assembly of A1/A2-dihydroxy-octyloxy pillar[5]arene 1,2-dichloroethane monosolvate

\section{Talal F. Al-Azemi, Mickey Vinodh, Abdirahman A. Mohamod and Fatemeh H. Alipour}

Computing details

Data collection: CrystalClear-SM Expert (Rigaku, 2009); cell refinement: CrystalClear-SM Expert (Rigaku, 2009); data reduction: CrystalStructure (Rigaku, 2010); program(s) used to solve structure: Il Milione (Burla et al., 2007); program(s) used to refine structure: SHELXL2017/1 (Sheldrick, 2015), ShelXle (Hübschle et al., 2011); molecular graphics: Mercury (Macrae et al., 2006).

1-(1,4-Dihydroxy)-2,3,4,5-(1,4-dioctyloxy)pillar[5]arene 1,2-dichloroethane solvent

Crystal data

$\mathrm{C}_{99} \mathrm{H}_{158} \mathrm{O}_{10} \cdot \mathrm{C}_{2} \mathrm{H}_{4} \mathrm{Cl}_{2}$

$M_{r}=1607.20$

Monoclinic, $C 2 / c$

$a=31.4629(12) \AA$

$b=20.2692(7) \AA$

$c=15.3703(11) \AA$

$\beta=91.275(6)^{\circ}$

$V=9799.7(9) \AA^{3}$

$Z=4$

Data collection

Rigaku R-AXIS RAPID diffractometer

Detector resolution: 10.000 pixels $\mathrm{mm}^{-1}$

$\omega$ scans

Absorption correction: multi-scan

(ABSCOR; Higashi, 1995)

$T_{\min }=0.774, T_{\max }=0.989$

42520 measured reflections

\section{Refinement}

Refinement on $F^{2}$

Least-squares matrix: full

$R\left[F^{2}>2 \sigma\left(F^{2}\right)\right]=0.049$

$w R\left(F^{2}\right)=0.137$

$S=1.08$

9959 reflections

528 parameters

24 restraints
$F(000)=3528$

$D_{\mathrm{x}}=1.089 \mathrm{Mg} \mathrm{m}^{-3}$

Mo $K \alpha$ radiation, $\lambda=0.71075 \AA$

Cell parameters from 11636 reflections

$\theta=3.1-26.3^{\circ}$

$\mu=0.12 \mathrm{~mm}^{-1}$

$T=150 \mathrm{~K}$

Block, colorless

$0.21 \times 0.13 \times 0.09 \mathrm{~mm}$

9959 independent reflections

7023 reflections with $I>2 \sigma(I)$

$R_{\text {int }}=0.032$

$\theta_{\text {max }}=26.3^{\circ}, \theta_{\min }=3.1^{\circ}$

$h=-37 \rightarrow 39$

$k=-25 \rightarrow 24$

$l=-19 \rightarrow 19$

Hydrogen site location: mixed

$\mathrm{H}$ atoms treated by a mixture of independent and constrained refinement

$w=1 /\left[\sigma^{2}\left(F_{\mathrm{o}}^{2}\right)+(0.064 P)^{2}+2.8339 P\right]$

where $P=\left(F_{\mathrm{o}}{ }^{2}+2 F_{\mathrm{c}}{ }^{2}\right) / 3$

$(\Delta / \sigma)_{\max }=0.001$

$\Delta \rho_{\max }=0.39 \mathrm{e} \AA^{-3}$

$\Delta \rho_{\min }=-0.43$ e $\AA^{-3}$ 


\section{Special details}

Geometry. All esds (except the esd in the dihedral angle between two 1.s. planes) are estimated using the full covariance matrix. The cell esds are taken into account individually in the estimation of esds in distances, angles and torsion angles; correlations between esds in cell parameters are only used when they are defined by crystal symmetry. An approximate (isotropic) treatment of cell esds is used for estimating esds involving l.s. planes.

Fractional atomic coordinates and isotropic or equivalent isotropic displacement parameters $\left(\AA^{2}\right)$

\begin{tabular}{|c|c|c|c|c|c|}
\hline & $x$ & $y$ & $z$ & $U_{\text {iso }} * / U_{\text {eq }}$ & Occ. $(<1)$ \\
\hline $\mathrm{O} 1$ & $0.52608(3)$ & $0.62986(5)$ & $0.52918(7)$ & $0.0395(3)$ & \\
\hline $\mathrm{Cl1}$ & $0.55186(2)$ & $0.76990(4)$ & $0.16233(5)$ & $0.0952(2)$ & \\
\hline C51A & $0.5073(3)$ & $0.7445(4)$ & $0.2239(6)$ & $0.125(2)$ & $0.464(7)$ \\
\hline $\mathrm{H} 51 \mathrm{~A}$ & 0.484105 & 0.759512 & 0.184099 & $0.150^{*}$ & $0.464(7)$ \\
\hline H51B & 0.508787 & 0.696374 & 0.213850 & $0.150^{*}$ & $0.464(7)$ \\
\hline C51B & $0.51449(18)$ & $0.7915(3)$ & $0.2421(5)$ & $0.123(2)$ & $0.536(7)$ \\
\hline $\mathrm{H} 51 \mathrm{C}$ & 0.527534 & 0.769901 & 0.293884 & $0.148^{*}$ & $0.536(7)$ \\
\hline H51D & 0.521354 & 0.838850 & 0.249897 & $0.148^{*}$ & $0.536(7)$ \\
\hline $\mathrm{O} 2$ & $0.64483(3)$ & $0.77669(5)$ & $0.35101(7)$ & $0.0433(3)$ & \\
\hline $\mathrm{O} 3$ & $0.51552(3)$ & $0.87675(5)$ & $0.51444(7)$ & $0.0392(3)$ & \\
\hline $\mathrm{O} 4$ & $0.58340(3)$ & $0.98033(5)$ & $0.21357(7)$ & $0.0407(3)$ & \\
\hline O5 & $0.47324(4)$ & $0.55783(7)$ & $0.42002(8)$ & $0.0535(3)$ & \\
\hline H5 & $0.4914(7)$ & $0.5732(14)$ & $0.4563(15)$ & $0.127(11)^{*}$ & \\
\hline $\mathrm{C} 1$ & $0.57574(4)$ & $0.63729(7)$ & $0.41632(10)$ & $0.0342(3)$ & \\
\hline $\mathrm{C} 2$ & $0.55453(4)$ & $0.66856(7)$ & $0.48322(10)$ & $0.0340(3)$ & \\
\hline $\mathrm{C} 3$ & $0.56248(4)$ & $0.73431(7)$ & $0.50300(10)$ & $0.0344(3)$ & \\
\hline $\mathrm{H} 3$ & 0.547151 & 0.754950 & 0.548068 & $0.041^{*}$ & \\
\hline $\mathrm{C} 4$ & $0.59246(4)$ & $0.77054(7)$ & $0.45812(10)$ & $0.0331(3)$ & \\
\hline $\mathrm{C} 5$ & $0.61468(4)$ & $0.73893(7)$ & $0.39254(10)$ & $0.0353(3)$ & \\
\hline C6 & $0.60609(4)$ & $0.67346(7)$ & $0.37175(10)$ & $0.0360(3)$ & \\
\hline H6 & 0.621183 & 0.652917 & 0.326262 & $0.043^{*}$ & \\
\hline $\mathrm{C} 7$ & $0.59975(5)$ & $0.84281(7)$ & $0.47991(10)$ & $0.0363(3)$ & \\
\hline $\mathrm{H} 7 \mathrm{~A}$ & 0.592112 & 0.850730 & 0.541169 & $0.044^{*}$ & \\
\hline H7B & 0.630313 & 0.853176 & 0.474216 & $0.044^{*}$ & \\
\hline $\mathrm{C} 8$ & $0.57387(4)$ & $0.88849(7)$ & $0.42128(10)$ & $0.0330(3)$ & \\
\hline C9 & $0.53189(4)$ & $0.90490(7)$ & $0.44047(10)$ & $0.0334(3)$ & \\
\hline $\mathrm{C} 10$ & $0.50904(5)$ & $0.94760(7)$ & $0.38618(10)$ & $0.0335(3)$ & \\
\hline H10 & 0.480914 & 0.959667 & 0.400799 & $0.040^{*}$ & \\
\hline C11 & $0.52661(4)$ & $0.97304(7)$ & $0.31073(10)$ & $0.0324(3)$ & \\
\hline $\mathrm{C} 12$ & $0.56792(4)$ & $0.95468(7)$ & $0.29032(10)$ & $0.0329(3)$ & \\
\hline $\mathrm{C} 13$ & $0.59133(4)$ & $0.91376(7)$ & $0.34602(10)$ & $0.0344(3)$ & \\
\hline H13 & 0.619778 & 0.902890 & 0.332339 & $0.041^{*}$ & \\
\hline $\mathrm{C} 14$ & 0.500000 & $1.01589(10)$ & 0.250000 & $0.0336(5)$ & \\
\hline $\mathrm{H} 14 \mathrm{~A}$ & 0.518806 & 1.044543 & 0.215644 & $0.040^{*}$ & 0.5 \\
\hline H14B & 0.481194 & 1.044544 & 0.284356 & $0.040^{*}$ & 0.5 \\
\hline $\mathrm{C} 15$ & $0.48802(5)$ & $0.56267(7)$ & $0.33650(10)$ & $0.0363(3)$ & \\
\hline $\mathrm{C} 16$ & $0.53117(5)$ & $0.56392(7)$ & $0.31800(10)$ & $0.0348(3)$ & \\
\hline $\mathrm{C} 17$ & $0.54243(5)$ & $0.56370(7)$ & $0.23073(10)$ & $0.0372(3)$ & \\
\hline H17 & 0.571688 & 0.564275 & 0.216727 & $0.045^{*}$ & \\
\hline
\end{tabular}




\begin{tabular}{|c|c|c|c|c|}
\hline $\mathrm{C} 18$ & $0.56560(5)$ & $0.56684(7)$ & $0.38916(10)$ & $0.0378(4)$ \\
\hline H18A & 0.556069 & 0.541780 & 0.440451 & $0.045^{*}$ \\
\hline H18B & 0.591725 & 0.545572 & 0.367930 & $0.045^{*}$ \\
\hline $\mathrm{C} 19$ & $0.49696(5)$ & $0.66342(7)$ & $0.58421(10)$ & $0.0384(3)$ \\
\hline H19A & 0.478500 & 0.693143 & 0.548950 & $0.046^{*}$ \\
\hline H19B & 0.512847 & 0.690397 & 0.627667 & $0.046^{*}$ \\
\hline $\mathrm{C} 20$ & $0.47014(5)$ & $0.61268(8)$ & $0.62986(11)$ & $0.0410(4)$ \\
\hline $\mathrm{H} 20 \mathrm{~A}$ & 0.488771 & 0.583358 & 0.665336 & $0.049^{*}$ \\
\hline H20B & 0.454848 & 0.585243 & 0.586033 & $0.049 *$ \\
\hline $\mathrm{C} 21$ & $0.43810(5)$ & $0.64623(7)$ & $0.68835(10)$ & 0.0387 (4) \\
\hline $\mathrm{H} 21 \mathrm{~A}$ & 0.416887 & 0.669869 & 0.651436 & $0.046^{*}$ \\
\hline $\mathrm{H} 21 \mathrm{~B}$ & 0.453135 & 0.679399 & 0.724939 & $0.046^{*}$ \\
\hline $\mathrm{C} 22$ & $0.41490(5)$ & $0.59824(8)$ & $0.74739(11)$ & $0.0410(4)$ \\
\hline $\mathrm{H} 22 \mathrm{~A}$ & 0.400150 & 0.564782 & 0.710783 & $0.049^{*}$ \\
\hline $\mathrm{H} 22 \mathrm{~B}$ & 0.436111 & 0.574949 & 0.784694 & $0.049^{*}$ \\
\hline $\mathrm{C} 23$ & $0.38255(5)$ & $0.63133(8)$ & $0.80523(10)$ & $0.0408(4)$ \\
\hline $\mathrm{H} 23 \mathrm{~A}$ & 0.358628 & 0.647936 & 0.768461 & $0.049 *$ \\
\hline H23B & 0.396056 & 0.669711 & 0.834528 & $0.049^{*}$ \\
\hline $\mathrm{C} 24$ & $0.36519(5)$ & $0.58494(8)$ & $0.87401(11)$ & $0.0440(4)$ \\
\hline $\mathrm{H} 24 \mathrm{~A}$ & 0.353325 & 0.545278 & 0.844775 & $0.053^{*}$ \\
\hline H24B & 0.388935 & 0.570446 & 0.912794 & $0.053^{*}$ \\
\hline $\mathrm{C} 25$ & $0.33098(6)$ & $0.61590(10)$ & $0.92887(12)$ & $0.0547(5)$ \\
\hline $\mathrm{H} 25 \mathrm{~A}$ & 0.306286 & 0.627359 & 0.890791 & $0.066^{*}$ \\
\hline $\mathrm{H} 25 \mathrm{~B}$ & 0.342120 & 0.657311 & 0.954821 & $0.066^{*}$ \\
\hline $\mathrm{C} 26$ & $0.31624(6)$ & $0.57082(11)$ & $1.00130(12)$ & $0.0643(6)$ \\
\hline $\mathrm{H} 26 \mathrm{~A}$ & 0.340499 & 0.559481 & 1.039500 & $0.077^{*}$ \\
\hline H26B & 0.304111 & 0.530447 & 0.975961 & $0.077^{*}$ \\
\hline $\mathrm{H} 26 \mathrm{C}$ & 0.294599 & 0.593494 & 1.035103 & $0.077^{*}$ \\
\hline $\mathrm{C} 27$ & $0.67085(5)$ & $0.74467(9)$ & $0.28883(11)$ & $0.0453(4)$ \\
\hline $\mathrm{H} 27 \mathrm{~A}$ & 0.652962 & 0.726534 & 0.240713 & $0.054^{*}$ \\
\hline H27B & 0.686720 & 0.707865 & 0.316701 & $0.054^{*}$ \\
\hline $\mathrm{C} 28$ & $0.70150(5)$ & $0.79524(9)$ & $0.25407(12)$ & $0.0503(4)$ \\
\hline $\mathrm{H} 28 \mathrm{~A}$ & 0.717227 & 0.815997 & 0.303391 & $0.060^{*}$ \\
\hline H28B & 0.685263 & 0.830239 & 0.223085 & $0.060^{*}$ \\
\hline $\mathrm{C} 29$ & $0.73314(5)$ & $0.76452(10)$ & $0.19214(12)$ & $0.0519(5)$ \\
\hline H29A & 0.750841 & 0.732111 & 0.224716 & $0.062 *$ \\
\hline H29B & 0.717211 & 0.740314 & 0.145944 & $0.062 *$ \\
\hline $\mathrm{C} 30$ & $0.76207(5)$ & $0.81448(10)$ & $0.14993(14)$ & $0.0607(5)$ \\
\hline H30A & 0.776793 & 0.840235 & 0.196288 & $0.073 *$ \\
\hline H30B & 0.744354 & 0.845558 & 0.115134 & $0.073 *$ \\
\hline $\mathrm{C} 31$ & $0.79542(5)$ & $0.78446(11)$ & $0.09109(13)$ & $0.0595(5)$ \\
\hline H31A & 0.815037 & 0.756882 & 0.126838 & $0.071 *$ \\
\hline H31B & 0.781035 & 0.755310 & 0.047959 & $0.071 *$ \\
\hline $\mathrm{C} 32$ & $0.82113(6)$ & $0.83584(11)$ & $0.04310(14)$ & $0.0687(6)$ \\
\hline $\mathrm{H} 32 \mathrm{~A}$ & 0.834554 & 0.865875 & 0.086439 & $0.082 *$ \\
\hline H32B & 0.801450 & 0.862480 & 0.006255 & $0.082 *$ \\
\hline C33 & $0.85581(6)$ & $0.80769(12)$ & $-0.01432(13)$ & $0.0695(6)$ \\
\hline H33A & 0.876784 & 0.783520 & 0.022559 & $0.083 *$ \\
\hline
\end{tabular}




\begin{tabular}{|c|c|c|c|c|}
\hline H33B & 0.842905 & 0.775904 & -0.056065 & $0.083 *$ \\
\hline $\mathrm{C} 34$ & $0.87843(7)$ & $0.86123(14)$ & $-0.06413(18)$ & $0.0942(9)$ \\
\hline H34A & 0.858974 & 0.879893 & -0.108306 & $0.113 *$ \\
\hline H34B & 0.903232 & 0.842378 & -0.092521 & $0.113 *$ \\
\hline $\mathrm{H} 34 \mathrm{C}$ & 0.887728 & 0.896067 & -0.023791 & $0.113^{*}$ \\
\hline $\mathrm{C} 35$ & $0.47133(5)$ & $0.88783(8)$ & $0.52980(10)$ & $0.0373(3)$ \\
\hline $\mathrm{H} 35 \mathrm{~A}$ & 0.454098 & 0.873954 & 0.478320 & $0.045^{*}$ \\
\hline H35B & 0.466222 & 0.935390 & 0.539873 & $0.045^{*}$ \\
\hline $\mathrm{C} 36$ & $0.45850(5)$ & $0.84864(8)$ & $0.60853(10)$ & $0.0409(4)$ \\
\hline H36A & 0.478914 & 0.857214 & 0.657167 & $0.049 *$ \\
\hline H36B & 0.459493 & 0.800958 & 0.594790 & $0.049 *$ \\
\hline $\mathrm{C} 37$ & $0.41377(5)$ & $0.86709(8)$ & $0.63631(11)$ & $0.0420(4)$ \\
\hline H37A & 0.413354 & 0.914617 & 0.651120 & $0.050 *$ \\
\hline H37B & 0.393878 & 0.860306 & 0.586303 & $0.050 *$ \\
\hline $\mathrm{C} 38$ & $0.39779(5)$ & $0.82805(8)$ & $0.71365(11)$ & $0.0427(4)$ \\
\hline H38A & 0.393628 & 0.781509 & 0.695872 & $0.051 *$ \\
\hline H38B & 0.419790 & 0.828902 & 0.760708 & $0.051 *$ \\
\hline C39 & $0.35635(5)$ & $0.85437(8)$ & $0.74903(11)$ & $0.0444(4)$ \\
\hline H39A & 0.361483 & 0.899063 & 0.773012 & $0.053^{*}$ \\
\hline H39B & 0.335450 & 0.858617 & 0.700247 & $0.053^{*}$ \\
\hline $\mathrm{C} 40$ & $0.33716(5)$ & $0.81172(8)$ & $0.81929(10)$ & $0.0399(4)$ \\
\hline $\mathrm{H} 40 \mathrm{~A}$ & 0.359749 & 0.800269 & 0.862559 & $0.048^{*}$ \\
\hline $\mathrm{H} 40 \mathrm{~B}$ & 0.326895 & 0.770091 & 0.792483 & $0.048^{*}$ \\
\hline $\mathrm{C} 41$ & $0.30058(5)$ & $0.84390(9)$ & $0.86639(12)$ & $0.0497(4)$ \\
\hline $\mathrm{H} 41 \mathrm{~A}$ & 0.310896 & 0.885039 & 0.894424 & $0.060 *$ \\
\hline H41B & 0.278109 & 0.856038 & 0.823211 & $0.060 *$ \\
\hline $\mathrm{C} 42$ & $0.28144(6)$ & $0.79983(11)$ & $0.93509(13)$ & $0.0605(5)$ \\
\hline $\mathrm{H} 42 \mathrm{~A}$ & 0.303696 & 0.786316 & 0.977014 & $0.073 *$ \\
\hline $\mathrm{H} 42 \mathrm{~B}$ & 0.269015 & 0.760640 & 0.907195 & $0.073 *$ \\
\hline $\mathrm{H} 42 \mathrm{C}$ & 0.259266 & 0.824090 & 0.965415 & $0.073 *$ \\
\hline $\mathrm{C} 43$ & $0.62086(5)$ & $0.95076(8)$ & 0.18009 (11) & 0.0402 (4) \\
\hline $\mathrm{H} 43 \mathrm{~A}$ & 0.617129 & 0.902359 & 0.175823 & $0.048^{*}$ \\
\hline $\mathrm{H} 43 \mathrm{~B}$ & 0.645483 & 0.959865 & 0.219482 & $0.048^{*}$ \\
\hline $\mathrm{C} 44$ & $0.62861(5)$ & $0.97944(8)$ & $0.09101(11)$ & $0.0419(4)$ \\
\hline $\mathrm{H} 44 \mathrm{~A}$ & 0.631473 & 1.027953 & 0.095763 & $0.050 *$ \\
\hline H44B & 0.603891 & 0.969841 & 0.052082 & $0.050 *$ \\
\hline $\mathrm{C} 45$ & $0.66860(5)$ & $0.95097(9)$ & $0.05176(11)$ & $0.0459(4)$ \\
\hline $\mathrm{H} 45 \mathrm{~A}$ & 0.664656 & 0.902882 & 0.043722 & $0.055^{*}$ \\
\hline $\mathrm{H} 45 \mathrm{~B}$ & 0.692667 & 0.957418 & 0.093479 & $0.055^{*}$ \\
\hline $\mathrm{C} 46$ & $0.68018(5)$ & $0.98138(9)$ & $-0.03522(11)$ & $0.0445(4)$ \\
\hline H46A & 0.656030 & 0.975589 & -0.076911 & $0.053 *$ \\
\hline H46B & 0.684781 & 1.029331 & -0.027189 & $0.053 *$ \\
\hline $\mathrm{C} 47$ & $0.71981(5)$ & $0.95118(9)$ & $-0.07369(11)$ & $0.0445(4)$ \\
\hline H47A & 0.714639 & 0.903585 & -0.083778 & $0.053 *$ \\
\hline H47B & 0.743532 & 0.955125 & -0.030597 & $0.053 *$ \\
\hline $\mathrm{C} 48$ & $0.73333(5)$ & $0.98252(9)$ & $-0.15878(11)$ & $0.0479(4)$ \\
\hline $\mathrm{H} 48 \mathrm{~A}$ & 0.709840 & 0.977884 & -0.202276 & $0.058^{*}$ \\
\hline $\mathrm{H} 48 \mathrm{~B}$ & 0.738095 & 1.030254 & -0.149018 & $0.058 *$ \\
\hline
\end{tabular}




\begin{tabular}{lllll} 
C49 & $0.77328(5)$ & $0.95275(10)$ & $-0.19575(12)$ & $0.0526(4)$ \\
H49A & 0.796653 & 0.956595 & -0.151846 & $0.063^{*}$ \\
H49B & 0.768337 & 0.905198 & -0.206667 & $0.063^{*}$ \\
C50 & $0.78709(7)$ & $0.98497(12)$ & $-0.27952(13)$ & $0.0736(6)$ \\
H50A & 0.764757 & 0.979396 & -0.324380 & $0.088^{*}$ \\
H50B & 0.813378 & 0.964245 & -0.298910 & $0.088^{*}$ \\
H50C & 0.792062 & 1.032112 & -0.269421 & $0.088^{*}$ \\
\hline
\end{tabular}

Atomic displacement parameters $\left(\AA^{2}\right)$

\begin{tabular}{|c|c|c|c|c|c|c|}
\hline & $U^{11}$ & $U^{22}$ & $U^{33}$ & $U^{12}$ & $U^{13}$ & $U^{23}$ \\
\hline $\mathrm{O} 1$ & $0.0483(6)$ & $0.0309(6)$ & $0.0401(6)$ & -0.0049 & $0.0156(5)$ & $0.0032(5)$ \\
\hline $\mathrm{Cl1}$ & $0.0912(4)$ & $0.0950(5)$ & $0.1005(5)$ & 0.0188 & $0.0283(4)$ & $0.0261(4)$ \\
\hline C51A & $0.138(5)$ & $0.078(4)$ & $0.163(6)$ & -0.007 (4) & $0.076(4)$ & -0.020 \\
\hline C51B & $0.133(5)$ & $0.076(4)$ & $0.164(5)$ & -0.035 & $0.068(4)$ & $-0.037(4)$ \\
\hline $\mathrm{O} 2$ & $0.0369(6)$ & $0.0438(6)$ & $0.0497(7)$ & -0.0064 & $0.0110(5)$ & 0.0078 \\
\hline $\mathrm{O} 3$ & $0.0412(6)$ & $0.0429(6)$ & $0.0337(6)$ & $-0.0041(4)$ & 0.0065 (4) & $0.0063(5)$ \\
\hline $\mathrm{O} 4$ & $0.0408(6)$ & $0.0406(6)$ & $0.0410(6)$ & $0.0016(4)$ & $0.0100(5)$ & $0.0117(5)$ \\
\hline O5 & $0.0490(7)$ & $0.0694(9)$ & $0.0427(7)$ & $-0.0088(6)$ & $0.0145(6)$ & $-0.0071(6)$ \\
\hline $\mathrm{C} 1$ & $0.0340(7)$ & $0.0327(8)$ & $0.0359(8)$ & $0.0015(6)$ & $0.0029(6)$ & $0.0063(6)$ \\
\hline $\mathrm{C} 2$ & $0.0352(8)$ & $0.0335(8)$ & $0.0335(8)$ & $-0.0037(6)$ & $0.0037(6)$ & $0.0079(6)$ \\
\hline $\mathrm{C} 3$ & $0.0372(8)$ & $0.0351(8)$ & $0.0309(8)$ & $-0.0017(6)$ & $0.0020(6)$ & $0.0049(6)$ \\
\hline $\mathrm{C} 4$ & $0.0327(7)$ & $0.0343(8)$ & $0.0321(8)$ & $-0.0029(6)$ & $-0.0050(6)$ & $0.0077(6)$ \\
\hline $\mathrm{C} 5$ & $0.0307(7)$ & $0.0389(9)$ & $0.0364(9)$ & $-0.0029(6)$ & $0.0012(6)$ & $0.0104(7)$ \\
\hline C6 & $0.0342(7)$ & $0.0385(9)$ & $0.0355(9)$ & $0.0019(6)$ & $0.0042(6)$ & $0.0053(7)$ \\
\hline $\mathrm{C} 7$ & $0.0369(8)$ & $0.0377(8)$ & $0.0341(8)$ & $-0.0090(6)$ & $-0.0030(6)$ & $0.0038(7)$ \\
\hline $\mathrm{C} 8$ & $0.0360(8)$ & $0.0297(8)$ & $0.0332(8)$ & $-0.0093(6)$ & $-0.0034(6)$ & $0.0009(6)$ \\
\hline C9 & $0.0409(8)$ & $0.0290(8)$ & $0.0303(8)$ & $-0.0088(6)$ & $0.0030(6)$ & $-0.0016(6)$ \\
\hline $\mathrm{C} 10$ & $0.0366(7)$ & $0.0289(8)$ & $0.0351(8)$ & $-0.0040(6)$ & $0.0038(6)$ & $-0.0032(6)$ \\
\hline C11 & $0.0388(8)$ & $0.0239(7)$ & $0.0344(8)$ & $-0.0052(5)$ & $0.0005(6)$ & $-0.0026(6)$ \\
\hline $\mathrm{C} 12$ & $0.0365(8)$ & $0.0293(8)$ & $0.0329(8)$ & $-0.0083(6)$ & $0.0031(6)$ & $0.0027(6)$ \\
\hline $\mathrm{C} 13$ & $0.0331(7)$ & $0.0325(8)$ & $0.0378(9)$ & $-0.0066(6)$ & $0.0008(6)$ & $0.0024(6)$ \\
\hline $\mathrm{C} 14$ & $0.0387(11)$ & $0.0260(10)$ & $0.0363(12)$ & 0.000 & $0.0047(9)$ & 0.000 \\
\hline $\mathrm{C} 15$ & $0.0422(8)$ & $0.0298(8)$ & $0.0374(9)$ & $-0.0028(6)$ & $0.0132(6)$ & $-0.0027(6)$ \\
\hline $\mathrm{C} 16$ & $0.0401(8)$ & $0.0230(7)$ & $0.0416(9)$ & $0.0008(6)$ & $0.0082(6)$ & $0.0019(6)$ \\
\hline $\mathrm{C} 17$ & $0.0349(8)$ & $0.0315(8)$ & $0.0456(10)$ & $0.0035(6)$ & $0.0114(6)$ & $0.0046(7)$ \\
\hline $\mathrm{C} 18$ & $0.0408(8)$ & $0.0315(8)$ & $0.0415(9)$ & $0.0028(6)$ & $0.0075(7)$ & $0.0046(7)$ \\
\hline C19 & $0.0472(9)$ & $0.0322(8)$ & $0.0363(9)$ & $-0.0031(6)$ & $0.0106(7)$ & $0.0005(7)$ \\
\hline $\mathrm{C} 20$ & $0.0501(9)$ & $0.0333(8)$ & $0.0402(9)$ & $-0.0041(7)$ & $0.0138(7)$ & $0.0022(7)$ \\
\hline $\mathrm{C} 21$ & $0.0466(9)$ & $0.0314(8)$ & $0.0385(9)$ & $-0.0034(6)$ & $0.0088(7)$ & $0.0015(7)$ \\
\hline $\mathrm{C} 22$ & $0.0463(9)$ & $0.0355(9)$ & $0.0418(9)$ & $-0.0043(6)$ & $0.0121(7)$ & $0.0004(7)$ \\
\hline $\mathrm{C} 23$ & $0.0440(9)$ & $0.0409(9)$ & $0.0380(9)$ & $-0.0034(7)$ & $0.0092(7)$ & $-0.0015(7)$ \\
\hline $\mathrm{C} 24$ & $0.0461(9)$ & $0.0485(10)$ & $0.0379(9)$ & $-0.0108(7)$ & $0.0096(7)$ & $-0.0024(7)$ \\
\hline $\mathrm{C} 25$ & $0.0503(10)$ & $0.0733(13)$ & $0.0409(10)$ & $-0.0048(9)$ & $0.0123(8)$ & $-0.0020(9)$ \\
\hline $\mathrm{C} 26$ & $0.0587(11)$ & $0.0906(16)$ & $0.0444(11)$ & $-0.0184(10)$ & $0.0169(9)$ & $-0.0040(10)$ \\
\hline $\mathrm{C} 27$ & $0.0373(8)$ & $0.0530(10)$ & $0.0460(10)$ & $-0.0031(7)$ & $0.0104(7)$ & $0.0058(8)$ \\
\hline $\mathrm{C} 28$ & $0.0367(8)$ & $0.0550(11)$ & $0.0595(12)$ & $-0.0036(7)$ & $0.0092(7)$ & $0.0170(9)$ \\
\hline $\mathrm{C} 29$ & $0.0385(9)$ & $0.0698(13)$ & $0.0477(11)$ & -0.0090 & $0.0064(7)$ & $0.0096(9)$ \\
\hline
\end{tabular}




\begin{tabular}{lllllll}
\hline C30 & $0.0396(9)$ & $0.0731(13)$ & $0.0700(14)$ & $0.0017(8)$ & $0.0155(9)$ & $0.0266(11)$ \\
C31 & $0.0412(9)$ & $0.0900(15)$ & $0.0475(11)$ & $-0.0105(9)$ & $0.0049(8)$ & $0.0104(10)$ \\
C32 & $0.0406(10)$ & $0.0929(16)$ & $0.0732(14)$ & $0.0043(9)$ & $0.0163(9)$ & $0.0312(12)$ \\
C33 & $0.0476(11)$ & $0.1114(18)$ & $0.0497(12)$ & $-0.0160(11)$ & $0.0077(9)$ & $0.0067(12)$ \\
C34 & $0.0600(13)$ & $0.125(2)$ & $0.099(2)$ & $0.0011(13)$ & $0.0372(13)$ & $0.0313(17)$ \\
C35 & $0.0415(8)$ & $0.0339(8)$ & $0.0366(9)$ & $-0.0028(6)$ & $0.0074(6)$ & $-0.0028(7)$ \\
C36 & $0.0473(9)$ & $0.0374(9)$ & $0.0385(9)$ & $-0.0047(7)$ & $0.0096(7)$ & $0.0006(7)$ \\
C37 & $0.0486(9)$ & $0.0350(9)$ & $0.0428(10)$ & $-0.0019(7)$ & $0.0111(7)$ & $-0.0003(7)$ \\
C38 & $0.0445(9)$ & $0.0442(10)$ & $0.0397(9)$ & $-0.0008(7)$ & $0.0079(7)$ & $0.0026(7)$ \\
C39 & $0.0488(9)$ & $0.0417(9)$ & $0.0432(10)$ & $-0.0013(7)$ & $0.0111(7)$ & $-0.0010(7)$ \\
C40 & $0.0376(8)$ & $0.0474(9)$ & $0.0350(9)$ & $-0.0037(7)$ & $0.0020(6)$ & $-0.0005(7)$ \\
C41 & $0.0451(9)$ & $0.0561(11)$ & $0.0484(11)$ & $-0.0038(8)$ & $0.0107(7)$ & $-0.0011(8)$ \\
C42 & $0.0487(10)$ & $0.0798(14)$ & $0.0538(12)$ & $-0.0054(9)$ & $0.0149(8)$ & $0.0059(10)$ \\
C43 & $0.0346(8)$ & $0.0426(9)$ & $0.0436(10)$ & $-0.0011(6)$ & $0.0062(6)$ & $0.0086(7)$ \\
C44 & $0.0400(8)$ & $0.0434(9)$ & $0.0427(10)$ & $-0.0030(7)$ & $0.0065(7)$ & $0.0094(7)$ \\
C45 & $0.0396(8)$ & $0.0524(10)$ & $0.0458(10)$ & $-0.0008(7)$ & $0.0071(7)$ & $0.0111(8)$ \\
C46 & $0.0416(9)$ & $0.0476(10)$ & $0.0446(10)$ & $-0.0028(7)$ & $0.0071(7)$ & $0.0076(8)$ \\
C47 & $0.0386(8)$ & $0.0490(10)$ & $0.0462(10)$ & $-0.0042(7)$ & $0.0065(7)$ & $0.0041(8)$ \\
C48 & $0.0458(9)$ & $0.0543(11)$ & $0.0441(10)$ & $-0.0056(7)$ & $0.0076(7)$ & $0.0013(8)$ \\
C49 & $0.0509(10)$ & $0.0589(11)$ & $0.0484(11)$ & $-0.0075(8)$ & $0.0107(8)$ & $-0.0092(9)$ \\
C50 & $0.0720(13)$ & $0.1006(18)$ & $0.0492(12)$ & $-0.0119(12)$ & $0.0220(10)$ & $-0.0087(12)$
\end{tabular}

Geometric parameters $\left(\AA,{ }^{\circ}\right)$

\begin{tabular}{llll}
\hline $\mathrm{O} 1-\mathrm{C} 2$ & $1.3942(16)$ & $\mathrm{C} 27-\mathrm{C} 28$ & $1.513(2)$ \\
$\mathrm{O} 1-\mathrm{C} 19$ & $1.4323(17)$ & $\mathrm{C} 27-\mathrm{H} 27 \mathrm{~A}$ & 0.9900 \\
$\mathrm{C} 11-\mathrm{C} 51 \mathrm{~B}$ & $1.772(5)$ & $\mathrm{C} 27-\mathrm{H} 27 \mathrm{~B}$ & 0.9900 \\
$\mathrm{C} 11-\mathrm{C} 51 \mathrm{~A}$ & $1.785(5)$ & $\mathrm{C} 28-\mathrm{C} 29$ & $1.525(2)$ \\
$\mathrm{C} 51 \mathrm{~A}-\mathrm{C} 51 \mathrm{~A}^{\mathrm{i}}$ & $0.932(11)$ & $\mathrm{C} 28-\mathrm{H} 28 \mathrm{~A}$ & 0.9900 \\
$\mathrm{C} 51 \mathrm{~A}-\mathrm{H} 51 \mathrm{~A}$ & 0.9900 & $\mathrm{C} 28-\mathrm{H} 28 \mathrm{~B}$ & 0.9900 \\
$\mathrm{C} 51 \mathrm{~A}-\mathrm{H} 51 \mathrm{~B}$ & 0.9900 & $\mathrm{C} 29-\mathrm{C} 30$ & $1.517(2)$ \\
$\mathrm{C} 51 \mathrm{~B}-\mathrm{C} 51 \mathrm{~B}^{\mathrm{i}}$ & $0.949(9)$ & $\mathrm{C} 29-\mathrm{H} 29 \mathrm{~A}$ & 0.9900 \\
$\mathrm{C} 51 \mathrm{~B}-\mathrm{H} 51 \mathrm{C}$ & 0.9900 & $\mathrm{C} 29-\mathrm{H} 29 \mathrm{~B}$ & 0.9900 \\
$\mathrm{C} 51 \mathrm{~B}-\mathrm{H} 51 \mathrm{D}$ & 0.9900 & $\mathrm{C} 30-\mathrm{C} 31$ & $1.527(3)$ \\
$\mathrm{O} 2-\mathrm{C} 5$ & $1.3857(17)$ & $\mathrm{C} 30-\mathrm{H} 30 \mathrm{~A}$ & 0.9900 \\
$\mathrm{O} 2-\mathrm{C} 27$ & $1.4280(19)$ & $\mathrm{C} 30-\mathrm{H} 30 \mathrm{~B}$ & 0.9900 \\
$\mathrm{O} 3-\mathrm{C} 9$ & $1.3817(17)$ & $\mathrm{C} 31-\mathrm{C} 32$ & $1.520(2)$ \\
$\mathrm{O} 3-\mathrm{C} 35$ & $1.4332(17)$ & $\mathrm{C} 31-\mathrm{H} 31 \mathrm{~A}$ & 0.9900 \\
$\mathrm{O} 4-\mathrm{C} 12$ & $1.3873(17)$ & $\mathrm{C} 31-\mathrm{H} 31 \mathrm{~B}$ & 0.9900 \\
$\mathrm{O} 4-\mathrm{C} 43$ & $1.4282(18)$ & $\mathrm{C} 32-\mathrm{C} 33$ & $1.529(3)$ \\
$\mathrm{O} 5-\mathrm{C} 15$ & $1.3786(19)$ & $\mathrm{C} 32-\mathrm{H} 32 \mathrm{~A}$ & 0.9900 \\
$\mathrm{O} 5-\mathrm{H} 5$ & $0.847(10)$ & $\mathrm{C} 32-\mathrm{H} 32 \mathrm{~B}$ & 0.9900 \\
$\mathrm{C} 1-\mathrm{C} 2$ & $1.391(2)$ & $\mathrm{C} 33-\mathrm{C} 34$ & $1.515(3)$ \\
$\mathrm{C} 1-\mathrm{C} 6$ & $1.3955(19)$ & $\mathrm{C} 33-\mathrm{H} 33 \mathrm{~A}$ & 0.9900 \\
$\mathrm{C} 1-\mathrm{C} 18$ & $1.520(2)$ & $\mathrm{C} 33-\mathrm{H} 33 \mathrm{~B}$ & 0.9900 \\
$\mathrm{C} 2-\mathrm{C} 3$ & $1.388(2)$ & $\mathrm{C} 34-\mathrm{H} 34 \mathrm{~A}$ & 0.9800 \\
$\mathrm{C} 3-\mathrm{C} 4$ & $1.3905(19)$ & $\mathrm{C} 34-\mathrm{H} 34 \mathrm{~B}$ & 0.9800 \\
$\mathrm{C} 3-\mathrm{H} 3$ & 0.9500 & $\mathrm{C} 34-\mathrm{H} 34 \mathrm{C}$ & 0.9800 \\
& & &
\end{tabular}




\begin{tabular}{|c|c|c|c|}
\hline $\mathrm{C} 4-\mathrm{C} 5$ & $1.395(2)$ & $\mathrm{C} 35-\mathrm{C} 36$ & $1.510(2)$ \\
\hline $\mathrm{C} 4-\mathrm{C} 7$ & $1.519(2)$ & $\mathrm{C} 35-\mathrm{H} 35 \mathrm{~A}$ & 0.9900 \\
\hline $\mathrm{C} 5-\mathrm{C} 6$ & $1.390(2)$ & C $35-\mathrm{H} 35 \mathrm{~B}$ & 0.9900 \\
\hline $\mathrm{C} 6-\mathrm{H} 6$ & 0.9500 & $\mathrm{C} 36-\mathrm{C} 37$ & $1.526(2)$ \\
\hline $\mathrm{C} 7-\mathrm{C} 8$ & $1.517(2)$ & $\mathrm{C} 36-\mathrm{H} 36 \mathrm{~A}$ & 0.9900 \\
\hline C7-H7A & 0.9900 & $\mathrm{C} 36-\mathrm{H} 36 \mathrm{~B}$ & 0.9900 \\
\hline C7-H7B & 0.9900 & $\mathrm{C} 37-\mathrm{C} 38$ & $1.523(2)$ \\
\hline $\mathrm{C} 8-\mathrm{C} 13$ & $1.389(2)$ & C37-H37A & 0.9900 \\
\hline $\mathrm{C} 8-\mathrm{C} 9$ & $1.400(2)$ & C37-H37B & 0.9900 \\
\hline $\mathrm{C} 9-\mathrm{C} 10$ & $1.391(2)$ & $\mathrm{C} 38-\mathrm{C} 39$ & $1.521(2)$ \\
\hline $\mathrm{C} 10-\mathrm{C} 11$ & $1.395(2)$ & $\mathrm{C} 38-\mathrm{H} 38 \mathrm{~A}$ & 0.9900 \\
\hline $\mathrm{C} 10-\mathrm{H} 10$ & 0.9500 & $\mathrm{C} 38-\mathrm{H} 38 \mathrm{~B}$ & 0.9900 \\
\hline $\mathrm{C} 11-\mathrm{C} 12$ & $1.395(2)$ & $\mathrm{C} 39-\mathrm{C} 40$ & $1.519(2)$ \\
\hline $\mathrm{C} 11-\mathrm{C} 14$ & $1.5139(18)$ & C39-H39A & 0.9900 \\
\hline $\mathrm{C} 12-\mathrm{C} 13$ & $1.391(2)$ & C39-H39B & 0.9900 \\
\hline C13-H13 & 0.9500 & $\mathrm{C} 40-\mathrm{C} 41$ & $1.520(2)$ \\
\hline $\mathrm{C} 14-\mathrm{H} 14 \mathrm{~A}$ & 0.9900 & $\mathrm{C} 40-\mathrm{H} 40 \mathrm{~A}$ & 0.9900 \\
\hline C14-H14B & 0.9900 & $\mathrm{C} 40-\mathrm{H} 40 \mathrm{~B}$ & 0.9900 \\
\hline $\mathrm{C} 15-\mathrm{C} 16$ & $1.394(2)$ & $\mathrm{C} 41-\mathrm{C} 42$ & $1.518(2)$ \\
\hline $\mathrm{C} 15-\mathrm{C} 17^{\mathrm{i}}$ & $1.394(2)$ & $\mathrm{C} 41-\mathrm{H} 41 \mathrm{~A}$ & 0.9900 \\
\hline $\mathrm{C} 16-\mathrm{C} 17$ & $1.395(2)$ & $\mathrm{C} 41-\mathrm{H} 41 \mathrm{~B}$ & 0.9900 \\
\hline $\mathrm{C} 16-\mathrm{C} 18$ & $1.523(2)$ & $\mathrm{C} 42-\mathrm{H} 42 \mathrm{~A}$ & 0.9800 \\
\hline $\mathrm{C} 17-\mathrm{H} 17$ & 0.9500 & $\mathrm{C} 42-\mathrm{H} 42 \mathrm{~B}$ & 0.9800 \\
\hline $\mathrm{C} 18-\mathrm{H} 18 \mathrm{~A}$ & 0.9900 & $\mathrm{C} 42-\mathrm{H} 42 \mathrm{C}$ & 0.9800 \\
\hline C18-H18B & 0.9900 & $\mathrm{C} 43-\mathrm{C} 44$ & $1.512(2)$ \\
\hline $\mathrm{C} 19-\mathrm{C} 20$ & $1.5128(19)$ & $\mathrm{C} 43-\mathrm{H} 43 \mathrm{~A}$ & 0.9900 \\
\hline C19-H19A & 0.9900 & $\mathrm{C} 43-\mathrm{H} 43 \mathrm{~B}$ & 0.9900 \\
\hline C19-H19B & 0.9900 & $\mathrm{C} 44-\mathrm{C} 45$ & $1.521(2)$ \\
\hline $\mathrm{C} 20-\mathrm{C} 21$ & $1.526(2)$ & $\mathrm{C} 44-\mathrm{H} 44 \mathrm{~A}$ & 0.9900 \\
\hline $\mathrm{C} 20-\mathrm{H} 20 \mathrm{~A}$ & 0.9900 & $\mathrm{C} 44-\mathrm{H} 44 \mathrm{~B}$ & 0.9900 \\
\hline $\mathrm{C} 20-\mathrm{H} 20 \mathrm{~B}$ & 0.9900 & $\mathrm{C} 45-\mathrm{C} 46$ & $1.524(2)$ \\
\hline $\mathrm{C} 21-\mathrm{C} 22$ & $1.527(2)$ & $\mathrm{C} 45-\mathrm{H} 45 \mathrm{~A}$ & 0.9900 \\
\hline $\mathrm{C} 21-\mathrm{H} 21 \mathrm{~A}$ & 0.9900 & $\mathrm{C} 45-\mathrm{H} 45 \mathrm{~B}$ & 0.9900 \\
\hline $\mathrm{C} 21-\mathrm{H} 21 \mathrm{~B}$ & 0.9900 & $\mathrm{C} 46-\mathrm{C} 47$ & $1.520(2)$ \\
\hline $\mathrm{C} 22-\mathrm{C} 23$ & $1.522(2)$ & $\mathrm{C} 46-\mathrm{H} 46 \mathrm{~A}$ & 0.9900 \\
\hline $\mathrm{C} 22-\mathrm{H} 22 \mathrm{~A}$ & 0.9900 & $\mathrm{C} 46-\mathrm{H} 46 \mathrm{~B}$ & 0.9900 \\
\hline $\mathrm{C} 22-\mathrm{H} 22 \mathrm{~B}$ & 0.9900 & $\mathrm{C} 47-\mathrm{C} 48$ & $1.523(2)$ \\
\hline $\mathrm{C} 23-\mathrm{C} 24$ & $1.525(2)$ & $\mathrm{C} 47-\mathrm{H} 47 \mathrm{~A}$ & 0.9900 \\
\hline $\mathrm{C} 23-\mathrm{H} 23 \mathrm{~A}$ & 0.9900 & $\mathrm{C} 47-\mathrm{H} 47 \mathrm{~B}$ & 0.9900 \\
\hline $\mathrm{C} 23-\mathrm{H} 23 \mathrm{~B}$ & 0.9900 & $\mathrm{C} 48-\mathrm{C} 49$ & $1.516(2)$ \\
\hline $\mathrm{C} 24-\mathrm{C} 25$ & $1.518(2)$ & $\mathrm{C} 48-\mathrm{H} 48 \mathrm{~A}$ & 0.9900 \\
\hline $\mathrm{C} 24-\mathrm{H} 24 \mathrm{~A}$ & 0.9900 & $\mathrm{C} 48-\mathrm{H} 48 \mathrm{~B}$ & 0.9900 \\
\hline $\mathrm{C} 24-\mathrm{H} 24 \mathrm{~B}$ & 0.9900 & $\mathrm{C} 49-\mathrm{C} 50$ & $1.516(3)$ \\
\hline $\mathrm{C} 25-\mathrm{C} 26$ & $1.521(3)$ & $\mathrm{C} 49-\mathrm{H} 49 \mathrm{~A}$ & 0.9900 \\
\hline $\mathrm{C} 25-\mathrm{H} 25 \mathrm{~A}$ & 0.9900 & $\mathrm{C} 49-\mathrm{H} 49 \mathrm{~B}$ & 0.9900 \\
\hline $\mathrm{C} 25-\mathrm{H} 25 \mathrm{~B}$ & 0.9900 & $\mathrm{C} 50-\mathrm{H} 50 \mathrm{~A}$ & 0.9800 \\
\hline $\mathrm{C} 26-\mathrm{H} 26 \mathrm{~A}$ & 0.9800 & $\mathrm{C} 50-\mathrm{H} 50 \mathrm{~B}$ & 0.9800 \\
\hline $\mathrm{C} 26-\mathrm{H} 26 \mathrm{~B}$ & 0.9800 & $\mathrm{C} 50-\mathrm{H} 50 \mathrm{C}$ & 0.9800 \\
\hline
\end{tabular}


C26-H26C $\quad 0.9800$

C2-O1-C19

$\mathrm{C} 51 \mathrm{~A}-\mathrm{C} 51 \mathrm{~A}-\mathrm{Cl1}$

C51A - C $51 \mathrm{~A}-\mathrm{H} 51 \mathrm{~A}$

$\mathrm{Cl1}-\mathrm{C} 51 \mathrm{~A}-\mathrm{H} 51 \mathrm{~A}$

C51A - C51A-H51B

$\mathrm{C} 11-\mathrm{C} 51 \mathrm{~A}-\mathrm{H} 51 \mathrm{~B}$

H51A-C51A-H51B

$\mathrm{C} 51 \mathrm{~B}^{\mathrm{i}}-\mathrm{C} 51 \mathrm{~B}-\mathrm{C} 11$

$\mathrm{C} 51 \mathrm{~B}-\mathrm{C} 51 \mathrm{~B}-\mathrm{H} 51 \mathrm{C}$

$\mathrm{C} 11-\mathrm{C} 51 \mathrm{~B}-\mathrm{H} 51 \mathrm{C}$

C51B $-\mathrm{C} 51 \mathrm{~B}-\mathrm{H} 51 \mathrm{D}$

Cl1-C51B-H51D

$\mathrm{H} 51 \mathrm{C}-\mathrm{C} 51 \mathrm{~B}-\mathrm{H} 51 \mathrm{D}$

$\mathrm{C} 5-\mathrm{O} 2-\mathrm{C} 27$

$\mathrm{C} 9-\mathrm{O} 3-\mathrm{C} 35$

$\mathrm{C} 12-\mathrm{O} 4-\mathrm{C} 43$

$\mathrm{C} 15-\mathrm{O} 5-\mathrm{H} 5$

$\mathrm{C} 2-\mathrm{C} 1-\mathrm{C} 6$

$\mathrm{C} 2-\mathrm{C} 1-\mathrm{C} 18$

$\mathrm{C} 6-\mathrm{C} 1-\mathrm{C} 18$

$\mathrm{C} 3-\mathrm{C} 2-\mathrm{C} 1$

$\mathrm{C} 3-\mathrm{C} 2-\mathrm{O} 1$

$\mathrm{C} 1-\mathrm{C} 2-\mathrm{O} 1$

$\mathrm{C} 2-\mathrm{C} 3-\mathrm{C} 4$

$\mathrm{C} 2-\mathrm{C} 3-\mathrm{H} 3$

$\mathrm{C} 4-\mathrm{C} 3-\mathrm{H} 3$

$\mathrm{C} 3-\mathrm{C} 4-\mathrm{C} 5$

$\mathrm{C} 3-\mathrm{C} 4-\mathrm{C} 7$

$\mathrm{C} 5-\mathrm{C} 4-\mathrm{C} 7$

$\mathrm{O} 2-\mathrm{C} 5-\mathrm{C} 6$

$\mathrm{O} 2-\mathrm{C} 5-\mathrm{C} 4$

$\mathrm{C} 6-\mathrm{C} 5-\mathrm{C} 4$

$\mathrm{C} 5-\mathrm{C} 6-\mathrm{C} 1$

$\mathrm{C} 5-\mathrm{C} 6-\mathrm{H} 6$

$\mathrm{C} 1-\mathrm{C} 6-\mathrm{H} 6$

$\mathrm{C} 8-\mathrm{C} 7-\mathrm{C} 4$

$\mathrm{C} 8-\mathrm{C} 7-\mathrm{H} 7 \mathrm{~A}$

$\mathrm{C} 4-\mathrm{C} 7-\mathrm{H} 7 \mathrm{~A}$

$\mathrm{C} 8-\mathrm{C} 7-\mathrm{H} 7 \mathrm{~B}$

$\mathrm{C} 4-\mathrm{C} 7-\mathrm{H} 7 \mathrm{~B}$

$\mathrm{H} 7 \mathrm{~A}-\mathrm{C} 7-\mathrm{H} 7 \mathrm{~B}$

C13-C $8-\mathrm{C} 9$

$\mathrm{C} 13-\mathrm{C} 8-\mathrm{C} 7$

$\mathrm{C} 9-\mathrm{C} 8-\mathrm{C} 7$

$\mathrm{O} 3-\mathrm{C} 9-\mathrm{C} 10$

$\mathrm{O} 3-\mathrm{C} 9-\mathrm{C} 8$
117.27 (11)

149.8 (14)

99.2

99.2

99.2

99.2

104.0

146.5 (11)

100.2

100.2

100.2

100.2

104.2

117.77 (12)

116.85 (12)

117.21 (11)

111 (2)

$117.92(14)$

$122.00(13)$

120.04 (13)

$120.80(13)$

122.95 (13)

116.24 (13)

121.29 (14)

119.4

119.4

118.17 (13)

120.05 (14)

121.77 (13)

123.57 (14)

$116.00(13)$

120.43 (13)

121.35 (14)

119.3

119.3

112.41 (12)

109.1

109.1

109.1

109.1

107.9

118.69 (14)

$120.19(13)$

121.10 (13)

123.59 (13)

116.49 (13)

\begin{tabular}{|c|c|}
\hline $\mathrm{C} 29-\mathrm{C} 28-\mathrm{H} 28 \mathrm{~A}$ & 109.2 \\
\hline $\mathrm{C} 27-\mathrm{C} 28-\mathrm{H} 28 \mathrm{~B}$ & 109.2 \\
\hline $\mathrm{C} 29-\mathrm{C} 28-\mathrm{H} 28 \mathrm{~B}$ & 109.2 \\
\hline $\mathrm{H} 28 \mathrm{~A}-\mathrm{C} 28-\mathrm{H} 28 \mathrm{~B}$ & 107.9 \\
\hline $\mathrm{C} 30-\mathrm{C} 29-\mathrm{C} 28$ & $113.61(16)$ \\
\hline $\mathrm{C} 30-\mathrm{C} 29-\mathrm{H} 29 \mathrm{~A}$ & 108.8 \\
\hline $\mathrm{C} 28-\mathrm{C} 29-\mathrm{H} 29 \mathrm{~A}$ & 108.8 \\
\hline $\mathrm{C} 30-\mathrm{C} 29-\mathrm{H} 29 \mathrm{~B}$ & 108.8 \\
\hline $\mathrm{C} 28-\mathrm{C} 29-\mathrm{H} 29 \mathrm{~B}$ & 108.8 \\
\hline $\mathrm{H} 29 \mathrm{~A}-\mathrm{C} 29-\mathrm{H} 29 \mathrm{~B}$ & 107.7 \\
\hline $\mathrm{C} 29-\mathrm{C} 30-\mathrm{C} 31$ & $114.46(17)$ \\
\hline $\mathrm{C} 29-\mathrm{C} 30-\mathrm{H} 30 \mathrm{~A}$ & 108.6 \\
\hline $\mathrm{C} 31-\mathrm{C} 30-\mathrm{H} 30 \mathrm{~A}$ & 108.6 \\
\hline $\mathrm{C} 29-\mathrm{C} 30-\mathrm{H} 30 \mathrm{~B}$ & 108.6 \\
\hline $\mathrm{C} 31-\mathrm{C} 30-\mathrm{H} 30 \mathrm{~B}$ & 108.6 \\
\hline $\mathrm{H} 30 \mathrm{~A}-\mathrm{C} 30-\mathrm{H} 30 \mathrm{~B}$ & 107.6 \\
\hline $\mathrm{C} 32-\mathrm{C} 31-\mathrm{C} 30$ & $113.23(18)$ \\
\hline $\mathrm{C} 32-\mathrm{C} 31-\mathrm{H} 31 \mathrm{~A}$ & 108.9 \\
\hline $\mathrm{C} 30-\mathrm{C} 31-\mathrm{H} 31 \mathrm{~A}$ & 108.9 \\
\hline $\mathrm{C} 32-\mathrm{C} 31-\mathrm{H} 31 \mathrm{~B}$ & 108.9 \\
\hline $\mathrm{C} 30-\mathrm{C} 31-\mathrm{H} 31 \mathrm{~B}$ & 108.9 \\
\hline $\mathrm{H} 31 \mathrm{~A}-\mathrm{C} 31-\mathrm{H} 31 \mathrm{~B}$ & 107.7 \\
\hline $\mathrm{C} 31-\mathrm{C} 32-\mathrm{C} 33$ & $114.76(19)$ \\
\hline $\mathrm{C} 31-\mathrm{C} 32-\mathrm{H} 32 \mathrm{~A}$ & 108.6 \\
\hline $\mathrm{C} 33-\mathrm{C} 32-\mathrm{H} 32 \mathrm{~A}$ & 108.6 \\
\hline $\mathrm{C} 31-\mathrm{C} 32-\mathrm{H} 32 \mathrm{~B}$ & 108.6 \\
\hline $\mathrm{C} 33-\mathrm{C} 32-\mathrm{H} 32 \mathrm{~B}$ & 108.6 \\
\hline $\mathrm{H} 32 \mathrm{~A}-\mathrm{C} 32-\mathrm{H} 32 \mathrm{~B}$ & 107.6 \\
\hline $\mathrm{C} 34-\mathrm{C} 33-\mathrm{C} 32$ & $111.9(2)$ \\
\hline $\mathrm{C} 34-\mathrm{C} 33-\mathrm{H} 33 \mathrm{~A}$ & 109.2 \\
\hline $\mathrm{C} 32-\mathrm{C} 33-\mathrm{H} 33 \mathrm{~A}$ & 109.2 \\
\hline $\mathrm{C} 34-\mathrm{C} 33-\mathrm{H} 33 \mathrm{~B}$ & 109.2 \\
\hline $\mathrm{C} 32-\mathrm{C} 33-\mathrm{H} 33 \mathrm{~B}$ & 109.2 \\
\hline $\mathrm{H} 33 \mathrm{~A}-\mathrm{C} 33-\mathrm{H} 33 \mathrm{~B}$ & 107.9 \\
\hline $\mathrm{C} 33-\mathrm{C} 34-\mathrm{H} 34 \mathrm{~A}$ & 109.5 \\
\hline $\mathrm{C} 33-\mathrm{C} 34-\mathrm{H} 34 \mathrm{~B}$ & 109.5 \\
\hline $\mathrm{H} 34 \mathrm{~A}-\mathrm{C} 34-\mathrm{H} 34 \mathrm{~B}$ & 109.5 \\
\hline $\mathrm{C} 33-\mathrm{C} 34-\mathrm{H} 34 \mathrm{C}$ & 109.5 \\
\hline $\mathrm{H} 34 \mathrm{~A}-\mathrm{C} 34-\mathrm{H} 34 \mathrm{C}$ & 109.5 \\
\hline $\mathrm{H} 34 \mathrm{~B}-\mathrm{C} 34-\mathrm{H} 34 \mathrm{C}$ & 109.5 \\
\hline $\mathrm{O} 3-\mathrm{C} 35-\mathrm{C} 36$ & $109.10(13)$ \\
\hline $\mathrm{O} 3-\mathrm{C} 35-\mathrm{H} 35 \mathrm{~A}$ & 109.9 \\
\hline $\mathrm{C} 36-\mathrm{C} 35-\mathrm{H} 35 \mathrm{~A}$ & 109.9 \\
\hline $\mathrm{O} 3-\mathrm{C} 35-\mathrm{H} 35 \mathrm{~B}$ & 109.9 \\
\hline $\mathrm{C} 36-\mathrm{C} 35-\mathrm{H} 35 \mathrm{~B}$ & 109.9 \\
\hline $\mathrm{H} 35 \mathrm{~A}-\mathrm{C} 35-\mathrm{H} 35 \mathrm{~B}$ & 108.3 \\
\hline
\end{tabular}




\begin{tabular}{|c|c|}
\hline $\mathrm{C} 10-\mathrm{C} 9-\mathrm{C} 8$ & $119.92(13)$ \\
\hline $\mathrm{C} 9-\mathrm{C} 10-\mathrm{C} 11$ & $121.32(13)$ \\
\hline $\mathrm{C} 9-\mathrm{C} 10-\mathrm{H} 10$ & 119.3 \\
\hline $\mathrm{C} 11-\mathrm{C} 10-\mathrm{H} 10$ & 119.3 \\
\hline $\mathrm{C} 10-\mathrm{C} 11-\mathrm{C} 12$ & $118.49(13)$ \\
\hline $\mathrm{C} 10-\mathrm{C} 11-\mathrm{C} 14$ & $120.06(12)$ \\
\hline $\mathrm{C} 12-\mathrm{C} 11-\mathrm{C} 14$ & $121.32(12)$ \\
\hline $\mathrm{O} 4-\mathrm{C} 12-\mathrm{C} 13$ & $123.76(13)$ \\
\hline $\mathrm{O} 4-\mathrm{C} 12-\mathrm{C} 11$ & $115.97(13)$ \\
\hline $\mathrm{C} 13-\mathrm{C} 12-\mathrm{C} 11$ & $120.26(13)$ \\
\hline $\mathrm{C} 8-\mathrm{C} 13-\mathrm{C} 12$ & $121.24(13)$ \\
\hline $\mathrm{C} 8-\mathrm{C} 13-\mathrm{H} 13$ & 119.4 \\
\hline $\mathrm{C} 12-\mathrm{C} 13-\mathrm{H} 13$ & 119.4 \\
\hline $\mathrm{C} 11-\mathrm{C} 14-\mathrm{C} 11^{\mathrm{i}}$ & $109.98(16)$ \\
\hline $\mathrm{C} 11-\mathrm{C} 14-\mathrm{H} 14 \mathrm{~A}$ & 109.7 \\
\hline $\mathrm{C} 11^{\mathrm{i}}-\mathrm{C} 14-\mathrm{H} 14 \mathrm{~A}$ & 109.7 \\
\hline $\mathrm{C} 11-\mathrm{C} 14-\mathrm{H} 14 \mathrm{~B}$ & 109.7 \\
\hline $\mathrm{C} 11^{\mathrm{i}}-\mathrm{C} 14-\mathrm{H} 14 \mathrm{~B}$ & 109.7 \\
\hline $\mathrm{H} 14 \mathrm{~A}-\mathrm{C} 14-\mathrm{H} 14 \mathrm{~B}$ & 108.2 \\
\hline $\mathrm{O} 5-\mathrm{C} 15-\mathrm{C} 16$ & 122.80 \\
\hline $\mathrm{O} 5-\mathrm{C} 15-\mathrm{C} 17^{\mathrm{i}}$ & $116.80(13)$ \\
\hline $\mathrm{C} 16-\mathrm{C} 15-\mathrm{C} 17^{\mathrm{i}}$ & 120.35 \\
\hline $\mathrm{C} 15-\mathrm{C} 16-\mathrm{C} 17$ & $117.74(14)$ \\
\hline $\mathrm{C} 15-\mathrm{C} 16-\mathrm{C} 18$ & $122.32(14)$ \\
\hline $\mathrm{C} 17-\mathrm{C} 16-\mathrm{C} 18$ & $119.93(13)$ \\
\hline $\mathrm{C} 15^{\mathrm{i}}-\mathrm{C} 17-\mathrm{C} 16$ & $121.87(13)$ \\
\hline $\mathrm{C} 15^{\mathrm{i}}-\mathrm{C} 17-\mathrm{H} 17$ & 119.1 \\
\hline $\mathrm{C} 16-\mathrm{C} 17-\mathrm{H} 17$ & 119.1 \\
\hline $\mathrm{C} 1-\mathrm{C} 18-\mathrm{C} 16$ & $112.06(12)$ \\
\hline $\mathrm{C} 1-\mathrm{C} 18-\mathrm{H} 18 \mathrm{~A}$ & 109.2 \\
\hline $\mathrm{C} 16-\mathrm{C} 18-\mathrm{H} 18 \mathrm{~A}$ & 109.2 \\
\hline $\mathrm{C} 1-\mathrm{C} 18-\mathrm{H} 18 \mathrm{~B}$ & 109.2 \\
\hline $\mathrm{C} 16-\mathrm{C} 18-\mathrm{H} 18 \mathrm{~B}$ & 109.2 \\
\hline $\mathrm{H} 18 \mathrm{~A}-\mathrm{C} 18-\mathrm{H} 18 \mathrm{~B}$ & 107.9 \\
\hline $\mathrm{O} 1-\mathrm{C} 19-\mathrm{C} 20$ & $108.77(12)$ \\
\hline $\mathrm{O} 1-\mathrm{C} 19-\mathrm{H} 19 \mathrm{~A}$ & 109.9 \\
\hline $\mathrm{C} 20-\mathrm{C} 19-\mathrm{H} 19 \mathrm{~A}$ & 109.9 \\
\hline $\mathrm{O} 1-\mathrm{C} 19-\mathrm{H} 19 \mathrm{~B}$ & 109.9 \\
\hline $\mathrm{C} 20-\mathrm{C} 19-\mathrm{H} 19 \mathrm{~B}$ & 109.9 \\
\hline $\mathrm{H} 19 \mathrm{~A}-\mathrm{C} 19-\mathrm{H} 19 \mathrm{~B}$ & 108.3 \\
\hline $\mathrm{C} 19-\mathrm{C} 20-\mathrm{C} 21$ & $110.68(12)$ \\
\hline $\mathrm{C} 19-\mathrm{C} 20-\mathrm{H} 20 \mathrm{~A}$ & 109.5 \\
\hline $\mathrm{C} 21-\mathrm{C} 20-\mathrm{H} 20 \mathrm{~A}$ & 109.5 \\
\hline $\mathrm{C} 19-\mathrm{C} 20-\mathrm{H} 20 \mathrm{~B}$ & 109.5 \\
\hline $\mathrm{C} 21-\mathrm{C} 20-\mathrm{H} 20 \mathrm{~B}$ & 109.5 \\
\hline $\mathrm{H} 20 \mathrm{~A}-\mathrm{C} 20-\mathrm{H} 20 \mathrm{~B}$ & 108.1 \\
\hline $\mathrm{C} 20-\mathrm{C} 21-\mathrm{C} 22$ & $113.40(12)$ \\
\hline $\mathrm{C} 20-\mathrm{C} 21-\mathrm{H} 21 \mathrm{~A}$ & 108.9 \\
\hline
\end{tabular}

\begin{tabular}{|c|c|}
\hline $\mathrm{C} 35-\mathrm{C} 36-\mathrm{C} 37$ & $111.09(13)$ \\
\hline $\mathrm{C} 35-\mathrm{C} 36-\mathrm{H} 36 \mathrm{~A}$ & 109.4 \\
\hline $\mathrm{C} 37-\mathrm{C} 36-\mathrm{H} 36 \mathrm{~A}$ & 109.4 \\
\hline $\mathrm{C} 35-\mathrm{C} 36-\mathrm{H} 36 \mathrm{~B}$ & 109.4 \\
\hline $\mathrm{C} 37-\mathrm{C} 36-\mathrm{H} 36 \mathrm{~B}$ & 109.4 \\
\hline $\mathrm{H} 36 \mathrm{~A}-\mathrm{C} 36-\mathrm{H} 36 \mathrm{~B}$ & 108.0 \\
\hline $\mathrm{C} 38-\mathrm{C} 37-\mathrm{C} 36$ & $114.43(14)$ \\
\hline $\mathrm{C} 38-\mathrm{C} 37-\mathrm{H} 37 \mathrm{~A}$ & 108.7 \\
\hline $\mathrm{C} 36-\mathrm{C} 37-\mathrm{H} 37 \mathrm{~A}$ & 108.7 \\
\hline $\mathrm{C} 38-\mathrm{C} 37-\mathrm{H} 37 \mathrm{~B}$ & 108.7 \\
\hline C $36-\mathrm{C} 37-\mathrm{H} 37 \mathrm{~B}$ & 108.7 \\
\hline $\mathrm{H} 37 \mathrm{~A}-\mathrm{C} 37-\mathrm{H} 37 \mathrm{~B}$ & 107.6 \\
\hline $\mathrm{C} 39-\mathrm{C} 38-\mathrm{C} 37$ & $113.42(14)$ \\
\hline $\mathrm{C} 39-\mathrm{C} 38-\mathrm{H} 38 \mathrm{~A}$ & 108.9 \\
\hline $\mathrm{C} 37-\mathrm{C} 38-\mathrm{H} 38 \mathrm{~A}$ & 108.9 \\
\hline $\mathrm{C} 39-\mathrm{C} 38-\mathrm{H} 38 \mathrm{~B}$ & 108.9 \\
\hline $\mathrm{C} 37-\mathrm{C} 38-\mathrm{H} 38 \mathrm{~B}$ & 108.9 \\
\hline $\mathrm{H} 38 \mathrm{~A}-\mathrm{C} 38-\mathrm{H} 38 \mathrm{~B}$ & 107.7 \\
\hline $\mathrm{C} 40-\mathrm{C} 39-\mathrm{C} 38$ & $114.34(14)$ \\
\hline $\mathrm{C} 40-\mathrm{C} 39-\mathrm{H} 39 \mathrm{~A}$ & 108.7 \\
\hline $\mathrm{C} 38-\mathrm{C} 39-\mathrm{H} 39 \mathrm{~A}$ & 108.7 \\
\hline $\mathrm{C} 40-\mathrm{C} 39-\mathrm{H} 39 \mathrm{~B}$ & 108.7 \\
\hline С $38-\mathrm{C} 39-\mathrm{H} 39 \mathrm{~B}$ & 108.7 \\
\hline $\mathrm{H} 39 \mathrm{~A}-\mathrm{C} 39-\mathrm{H} 39 \mathrm{~B}$ & 107.6 \\
\hline $\mathrm{C} 39-\mathrm{C} 40-\mathrm{C} 41$ & $114.30(14)$ \\
\hline $\mathrm{C} 39-\mathrm{C} 40-\mathrm{H} 40 \mathrm{~A}$ & 108.7 \\
\hline $\mathrm{C} 41-\mathrm{C} 40-\mathrm{H} 40 \mathrm{~A}$ & 108.7 \\
\hline $\mathrm{C} 39-\mathrm{C} 40-\mathrm{H} 40 \mathrm{~B}$ & 108.7 \\
\hline $\mathrm{C} 41-\mathrm{C} 40-\mathrm{H} 40 \mathrm{~B}$ & 108.7 \\
\hline $\mathrm{H} 40 \mathrm{~A}-\mathrm{C} 40-\mathrm{H} 40 \mathrm{~B}$ & 107.6 \\
\hline $\mathrm{C} 42-\mathrm{C} 41-\mathrm{C} 40$ & $113.27(15)$ \\
\hline $\mathrm{C} 42-\mathrm{C} 41-\mathrm{H} 41 \mathrm{~A}$ & 108.9 \\
\hline $\mathrm{C} 40-\mathrm{C} 41-\mathrm{H} 41 \mathrm{~A}$ & 108.9 \\
\hline $\mathrm{C} 42-\mathrm{C} 41-\mathrm{H} 41 \mathrm{~B}$ & 108.9 \\
\hline $\mathrm{C} 40-\mathrm{C} 41-\mathrm{H} 41 \mathrm{~B}$ & 108.9 \\
\hline $\mathrm{H} 41 \mathrm{~A}-\mathrm{C} 41-\mathrm{H} 41 \mathrm{~B}$ & 107.7 \\
\hline $\mathrm{C} 41-\mathrm{C} 42-\mathrm{H} 42 \mathrm{~A}$ & 109.5 \\
\hline $\mathrm{C} 41-\mathrm{C} 42-\mathrm{H} 42 \mathrm{~B}$ & 109.5 \\
\hline $\mathrm{H} 42 \mathrm{~A}-\mathrm{C} 42-\mathrm{H} 42 \mathrm{~B}$ & 109.5 \\
\hline $\mathrm{C} 41-\mathrm{C} 42-\mathrm{H} 42 \mathrm{C}$ & 109.5 \\
\hline $\mathrm{H} 42 \mathrm{~A}-\mathrm{C} 42-\mathrm{H} 42 \mathrm{C}$ & 109.5 \\
\hline $\mathrm{H} 42 \mathrm{~B}-\mathrm{C} 42-\mathrm{H} 42 \mathrm{C}$ & 109.5 \\
\hline $\mathrm{O} 4-\mathrm{C} 43-\mathrm{C} 44$ & $108.42(12)$ \\
\hline $\mathrm{O} 4-\mathrm{C} 43-\mathrm{H} 43 \mathrm{~A}$ & 110.0 \\
\hline $\mathrm{C} 44-\mathrm{C} 43-\mathrm{H} 43 \mathrm{~A}$ & 110.0 \\
\hline $\mathrm{O} 4-\mathrm{C} 43-\mathrm{H} 43 \mathrm{~B}$ & 110.0 \\
\hline $\mathrm{C} 44-\mathrm{C} 43-\mathrm{H} 43 \mathrm{~B}$ & 110.0 \\
\hline $\mathrm{H} 43 \mathrm{~A}-\mathrm{C} 43-\mathrm{H} 43 \mathrm{~B}$ & 108.4 \\
\hline
\end{tabular}




\begin{tabular}{|c|c|c|c|}
\hline $\mathrm{C} 22-\mathrm{C} 21-\mathrm{H} 21 \mathrm{~A}$ & 108.9 & $\mathrm{C} 43-\mathrm{C} 44-\mathrm{C} 45$ & $111.40(13)$ \\
\hline $\mathrm{C} 20-\mathrm{C} 21-\mathrm{H} 21 \mathrm{~B}$ & 108.9 & $\mathrm{C} 43-\mathrm{C} 44-\mathrm{H} 44 \mathrm{~A}$ & 109.3 \\
\hline $\mathrm{C} 22-\mathrm{C} 21-\mathrm{H} 21 \mathrm{~B}$ & 108.9 & $\mathrm{C} 45-\mathrm{C} 44-\mathrm{H} 44 \mathrm{~A}$ & 109.3 \\
\hline $\mathrm{H} 21 \mathrm{~A}-\mathrm{C} 21-\mathrm{H} 21 \mathrm{~B}$ & 107.7 & $\mathrm{C} 43-\mathrm{C} 44-\mathrm{H} 44 \mathrm{~B}$ & 109.3 \\
\hline $\mathrm{C} 23-\mathrm{C} 22-\mathrm{C} 21$ & $113.65(13)$ & $\mathrm{C} 45-\mathrm{C} 44-\mathrm{H} 44 \mathrm{~B}$ & 109.3 \\
\hline $\mathrm{C} 23-\mathrm{C} 22-\mathrm{H} 22 \mathrm{~A}$ & 108.8 & $\mathrm{H} 44 \mathrm{~A}-\mathrm{C} 44-\mathrm{H} 44 \mathrm{~B}$ & 108.0 \\
\hline $\mathrm{C} 21-\mathrm{C} 22-\mathrm{H} 22 \mathrm{~A}$ & 108.8 & $\mathrm{C} 44-\mathrm{C} 45-\mathrm{C} 46$ & $114.25(14)$ \\
\hline $\mathrm{C} 23-\mathrm{C} 22-\mathrm{H} 22 \mathrm{~B}$ & 108.8 & $\mathrm{C} 44-\mathrm{C} 45-\mathrm{H} 45 \mathrm{~A}$ & 108.7 \\
\hline $\mathrm{C} 21-\mathrm{C} 22-\mathrm{H} 22 \mathrm{~B}$ & 108.8 & $\mathrm{C} 46-\mathrm{C} 45-\mathrm{H} 45 \mathrm{~A}$ & 108.7 \\
\hline $\mathrm{H} 22 \mathrm{~A}-\mathrm{C} 22-\mathrm{H} 22 \mathrm{~B}$ & 107.7 & $\mathrm{C} 44-\mathrm{C} 45-\mathrm{H} 45 \mathrm{~B}$ & 108.7 \\
\hline $\mathrm{C} 22-\mathrm{C} 23-\mathrm{C} 24$ & $112.81(13)$ & $\mathrm{C} 46-\mathrm{C} 45-\mathrm{H} 45 \mathrm{~B}$ & 108.7 \\
\hline $\mathrm{C} 22-\mathrm{C} 23-\mathrm{H} 23 \mathrm{~A}$ & 109.0 & $\mathrm{H} 45 \mathrm{~A}-\mathrm{C} 45-\mathrm{H} 45 \mathrm{~B}$ & 107.6 \\
\hline $\mathrm{C} 24-\mathrm{C} 23-\mathrm{H} 23 \mathrm{~A}$ & 109.0 & $\mathrm{C} 47-\mathrm{C} 46-\mathrm{C} 45$ & $113.11(14)$ \\
\hline $\mathrm{C} 22-\mathrm{C} 23-\mathrm{H} 23 \mathrm{~B}$ & 109.0 & $\mathrm{C} 47-\mathrm{C} 46-\mathrm{H} 46 \mathrm{~A}$ & 109.0 \\
\hline $\mathrm{C} 24-\mathrm{C} 23-\mathrm{H} 23 \mathrm{~B}$ & 109.0 & $\mathrm{C} 45-\mathrm{C} 46-\mathrm{H} 46 \mathrm{~A}$ & 109.0 \\
\hline $\mathrm{H} 23 \mathrm{~A}-\mathrm{C} 23-\mathrm{H} 23 \mathrm{~B}$ & 107.8 & $\mathrm{C} 47-\mathrm{C} 46-\mathrm{H} 46 \mathrm{~B}$ & 109.0 \\
\hline $\mathrm{C} 25-\mathrm{C} 24-\mathrm{C} 23$ & $113.55(15)$ & $\mathrm{C} 45-\mathrm{C} 46-\mathrm{H} 46 \mathrm{~B}$ & 109.0 \\
\hline $\mathrm{C} 25-\mathrm{C} 24-\mathrm{H} 24 \mathrm{~A}$ & 108.9 & $\mathrm{H} 46 \mathrm{~A}-\mathrm{C} 46-\mathrm{H} 46 \mathrm{~B}$ & 107.8 \\
\hline $\mathrm{C} 23-\mathrm{C} 24-\mathrm{H} 24 \mathrm{~A}$ & 108.9 & $\mathrm{C} 46-\mathrm{C} 47-\mathrm{C} 48$ & $114.40(14)$ \\
\hline $\mathrm{C} 25-\mathrm{C} 24-\mathrm{H} 24 \mathrm{~B}$ & 108.9 & $\mathrm{C} 46-\mathrm{C} 47-\mathrm{H} 47 \mathrm{~A}$ & 108.7 \\
\hline $\mathrm{C} 23-\mathrm{C} 24-\mathrm{H} 24 \mathrm{~B}$ & 108.9 & $\mathrm{C} 48-\mathrm{C} 47-\mathrm{H} 47 \mathrm{~A}$ & 108.7 \\
\hline $\mathrm{H} 24 \mathrm{~A}-\mathrm{C} 24-\mathrm{H} 24 \mathrm{~B}$ & 107.7 & $\mathrm{C} 46-\mathrm{C} 47-\mathrm{H} 47 \mathrm{~B}$ & 108.7 \\
\hline $\mathrm{C} 24-\mathrm{C} 25-\mathrm{C} 26$ & $112.95(17)$ & $\mathrm{C} 48-\mathrm{C} 47-\mathrm{H} 47 \mathrm{~B}$ & 108.7 \\
\hline $\mathrm{C} 24-\mathrm{C} 25-\mathrm{H} 25 \mathrm{~A}$ & 109.0 & $\mathrm{H} 47 \mathrm{~A}-\mathrm{C} 47-\mathrm{H} 47 \mathrm{~B}$ & 107.6 \\
\hline $\mathrm{C} 26-\mathrm{C} 25-\mathrm{H} 25 \mathrm{~A}$ & 109.0 & $\mathrm{C} 49-\mathrm{C} 48-\mathrm{C} 47$ & $113.93(15)$ \\
\hline $\mathrm{C} 24-\mathrm{C} 25-\mathrm{H} 25 \mathrm{~B}$ & 109.0 & $\mathrm{C} 49-\mathrm{C} 48-\mathrm{H} 48 \mathrm{~A}$ & 108.8 \\
\hline $\mathrm{C} 26-\mathrm{C} 25-\mathrm{H} 25 \mathrm{~B}$ & 109.0 & $\mathrm{C} 47-\mathrm{C} 48-\mathrm{H} 48 \mathrm{~A}$ & 108.8 \\
\hline $\mathrm{H} 25 \mathrm{~A}-\mathrm{C} 25-\mathrm{H} 25 \mathrm{~B}$ & 107.8 & $\mathrm{C} 49-\mathrm{C} 48-\mathrm{H} 48 \mathrm{~B}$ & 108.8 \\
\hline $\mathrm{C} 25-\mathrm{C} 26-\mathrm{H} 26 \mathrm{~A}$ & 109.5 & $\mathrm{C} 47-\mathrm{C} 48-\mathrm{H} 48 \mathrm{~B}$ & 108.8 \\
\hline $\mathrm{C} 25-\mathrm{C} 26-\mathrm{H} 26 \mathrm{~B}$ & 109.5 & $\mathrm{H} 48 \mathrm{~A}-\mathrm{C} 48-\mathrm{H} 48 \mathrm{~B}$ & 107.7 \\
\hline $\mathrm{H} 26 \mathrm{~A}-\mathrm{C} 26-\mathrm{H} 26 \mathrm{~B}$ & 109.5 & $\mathrm{C} 50-\mathrm{C} 49-\mathrm{C} 48$ & $113.74(17)$ \\
\hline $\mathrm{C} 25-\mathrm{C} 26-\mathrm{H} 26 \mathrm{C}$ & 109.5 & $\mathrm{C} 50-\mathrm{C} 49-\mathrm{H} 49 \mathrm{~A}$ & 108.8 \\
\hline $\mathrm{H} 26 \mathrm{~A}-\mathrm{C} 26-\mathrm{H} 26 \mathrm{C}$ & 109.5 & $\mathrm{C} 48-\mathrm{C} 49-\mathrm{H} 49 \mathrm{~A}$ & 108.8 \\
\hline $\mathrm{H} 26 \mathrm{~B}-\mathrm{C} 26-\mathrm{H} 26 \mathrm{C}$ & 109.5 & $\mathrm{C} 50-\mathrm{C} 49-\mathrm{H} 49 \mathrm{~B}$ & 108.8 \\
\hline $\mathrm{O} 2-\mathrm{C} 27-\mathrm{C} 28$ & $107.93(14)$ & $\mathrm{C} 48-\mathrm{C} 49-\mathrm{H} 49 \mathrm{~B}$ & 108.8 \\
\hline $\mathrm{O} 2-\mathrm{C} 27-\mathrm{H} 27 \mathrm{~A}$ & 110.1 & $\mathrm{H} 49 \mathrm{~A}-\mathrm{C} 49-\mathrm{H} 49 \mathrm{~B}$ & 107.7 \\
\hline $\mathrm{C} 28-\mathrm{C} 27-\mathrm{H} 27 \mathrm{~A}$ & 110.1 & $\mathrm{C} 49-\mathrm{C} 50-\mathrm{H} 50 \mathrm{~A}$ & 109.5 \\
\hline $\mathrm{O} 2-\mathrm{C} 27-\mathrm{H} 27 \mathrm{~B}$ & 110.1 & $\mathrm{C} 49-\mathrm{C} 50-\mathrm{H} 50 \mathrm{~B}$ & 109.5 \\
\hline $\mathrm{C} 28-\mathrm{C} 27-\mathrm{H} 27 \mathrm{~B}$ & 110.1 & $\mathrm{H} 50 \mathrm{~A}-\mathrm{C} 50-\mathrm{H} 50 \mathrm{~B}$ & 109.5 \\
\hline $\mathrm{H} 27 \mathrm{~A}-\mathrm{C} 27-\mathrm{H} 27 \mathrm{~B}$ & 108.4 & $\mathrm{C} 49-\mathrm{C} 50-\mathrm{H} 50 \mathrm{C}$ & 109.5 \\
\hline $\mathrm{C} 27-\mathrm{C} 28-\mathrm{C} 29$ & $111.95(15)$ & $\mathrm{H} 50 \mathrm{~A}-\mathrm{C} 50-\mathrm{H} 50 \mathrm{C}$ & 109.5 \\
\hline $\mathrm{C} 27-\mathrm{C} 28-\mathrm{H} 28 \mathrm{~A}$ & 109.2 & $\mathrm{H} 50 \mathrm{~B}-\mathrm{C} 50-\mathrm{H} 50 \mathrm{C}$ & 109.5 \\
\hline $\mathrm{C} 6-\mathrm{C} 1-\mathrm{C} 2-\mathrm{C} 3$ & $1.9(2)$ & $\mathrm{O} 4-\mathrm{C} 12-\mathrm{C} 13-\mathrm{C} 8$ & $-178.75(13)$ \\
\hline $\mathrm{C} 18-\mathrm{C} 1-\mathrm{C} 2-\mathrm{C} 3$ & $-175.80(14)$ & $\mathrm{C} 11-\mathrm{C} 12-\mathrm{C} 13-\mathrm{C} 8$ & $2.2(2)$ \\
\hline $\mathrm{C} 6-\mathrm{C} 1-\mathrm{C} 2-\mathrm{O} 1$ & $-176.77(12)$ & $\mathrm{C} 10-\mathrm{C} 11-\mathrm{C} 14-\mathrm{C} 11^{\mathrm{i}}$ & $-83.32(12)$ \\
\hline $\mathrm{C} 18-\mathrm{C} 1-\mathrm{C} 2-\mathrm{O} 1$ & $5.5(2)$ & $\mathrm{C} 12-\mathrm{C} 11-\mathrm{C} 14-\mathrm{C} 11^{\mathrm{i}}$ & $92.57(13)$ \\
\hline $\mathrm{C} 19-\mathrm{O} 1-\mathrm{C} 2-\mathrm{C} 3$ & $15.2(2)$ & $\mathrm{O} 5-\mathrm{C} 15-\mathrm{C} 16-\mathrm{C} 17$ & $175.06(13)$ \\
\hline
\end{tabular}




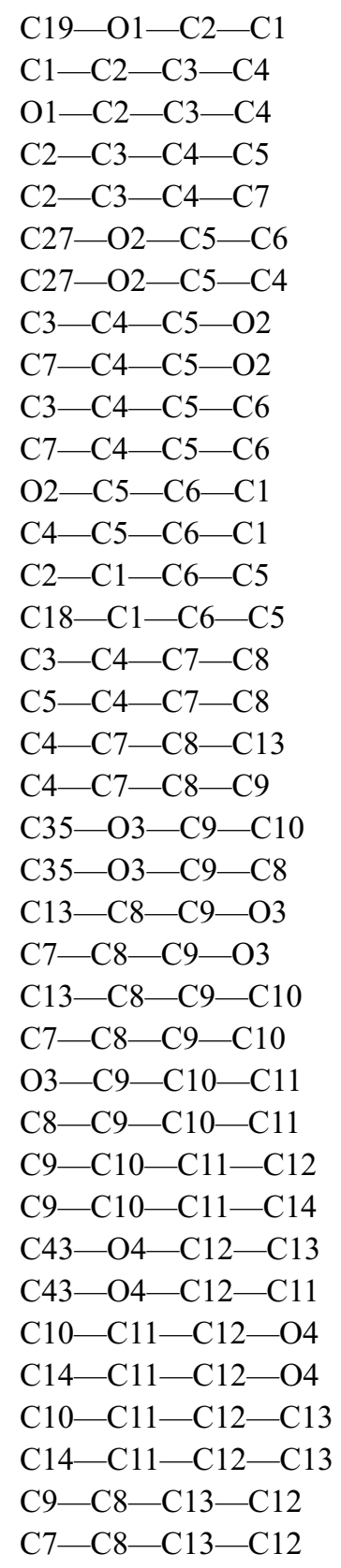

$\begin{array}{ll}-166.12(13) & \mathrm{C} 17-\mathrm{C} 15-\mathrm{C} 16-\mathrm{C} 17 \\ -1.4(2) & \mathrm{O} 5-\mathrm{C} 15-\mathrm{C} 16-\mathrm{C} 18 \\ 177.16(13) & \mathrm{C} 17-\mathrm{C} 15-\mathrm{C} 16-\mathrm{C} 18 \\ -0.3(2) & \mathrm{C} 15-\mathrm{C} 16-\mathrm{C} 17-\mathrm{C} 15^{\mathrm{i}} \\ 178.65(13) & \mathrm{C} 18-\mathrm{C} 16-\mathrm{C} 17-\mathrm{C} 15^{\mathrm{i}} \\ -5.6(2) & \mathrm{C} 2-\mathrm{C} 1-\mathrm{C} 18-\mathrm{C} 16 \\ 174.64(13) & \mathrm{C} 6-\mathrm{C} 1-\mathrm{C} 18-\mathrm{C} 16 \\ -178.67(12) & \mathrm{C} 15-\mathrm{C} 16-\mathrm{C} 18-\mathrm{C} 1 \\ 2.4(2) & \mathrm{C} 17-\mathrm{C} 16-\mathrm{C} 18-\mathrm{C} 1 \\ 1.5(2) & \mathrm{C} 2-\mathrm{O} 1-\mathrm{C} 19-\mathrm{C} 20 \\ -177.41(13) & \mathrm{O} 1-\mathrm{C} 19-\mathrm{C} 20-\mathrm{C} 21 \\ 179.18(13) & \mathrm{C} 19-\mathrm{C} 20-\mathrm{C} 21-\mathrm{C} 22 \\ -1.0(2) & \mathrm{C} 20-\mathrm{C} 21-\mathrm{C} 22-\mathrm{C} 23 \\ -0.7(2) & \mathrm{C} 21-\mathrm{C} 22-\mathrm{C} 23-\mathrm{C} 24 \\ 177.07(13) & \mathrm{C} 22-\mathrm{C} 23-\mathrm{C} 24-\mathrm{C} 25 \\ -94.67(16) & \mathrm{C} 23-\mathrm{C} 24-\mathrm{C} 25-\mathrm{C} 26 \\ 84.26(17) & \mathrm{C} 5-\mathrm{O} 2-\mathrm{C} 27-\mathrm{C} 28 \\ -92.92(16) & \mathrm{O} 2-\mathrm{C} 27-\mathrm{C} 28-\mathrm{C} 29 \\ 85.65(17) & \mathrm{C} 27-\mathrm{C} 28-\mathrm{C} 29-\mathrm{C} 30 \\ 6.3(2) & \mathrm{C} 28-\mathrm{C} 29-\mathrm{C} 30-\mathrm{C} 31 \\ -173.83(12) & \mathrm{C} 29-\mathrm{C} 30-\mathrm{C} 31-\mathrm{C} 32 \\ 177.87(12) & \mathrm{C} 30-\mathrm{C} 31-\mathrm{C} 32-\mathrm{C} 33 \\ -0.7(2) & \mathrm{C} 31-\mathrm{C} 32-\mathrm{C} 33-\mathrm{C} 34 \\ -2.2(2) & \mathrm{C} 9-\mathrm{O} 3-\mathrm{C} 35-\mathrm{C} 36 \\ 179.17(13) & \mathrm{O} 3-\mathrm{C} 35-\mathrm{C} 36-\mathrm{C} 37 \\ -177.94(13) & \mathrm{C} 35-\mathrm{C} 36-\mathrm{C} 37-\mathrm{C} 38 \\ 2.2(2) & \mathrm{C} 36-\mathrm{C} 37-\mathrm{C} 38-\mathrm{C} 39 \\ 0.1(2) & \mathrm{C} 37-\mathrm{C} 38-\mathrm{C} 39-\mathrm{C} 40 \\ 176.09(13) & \mathrm{C} 38-\mathrm{C} 39-\mathrm{C} 40-\mathrm{C} 41 \\ 15.6(2) & \mathrm{C} 39-\mathrm{C} 40-\mathrm{C} 41-\mathrm{C} 42 \\ -165.35(12) & \mathrm{C} 12-\mathrm{O} 4-\mathrm{C} 43-\mathrm{C} 44 \\ 178.63(12) & \mathrm{O} 4-\mathrm{C} 43-\mathrm{C} 44-\mathrm{C} 45 \\ 2.7(2) & \mathrm{C} 43-\mathrm{C} 44-\mathrm{C} 45-\mathrm{C} 46 \\ -2.2(2) & \mathrm{C} 44-\mathrm{C} 45-\mathrm{C} 46-\mathrm{C} 47 \\ -178.20(13) & \mathrm{C} 45-\mathrm{C} 46-\mathrm{C} 47-\mathrm{C} 48 \\ 0.1(2) & \mathrm{C} 46-\mathrm{C} 47-\mathrm{C} 48-\mathrm{C} 49 \\ 178.69(13) & \mathrm{C} 47-\mathrm{C} 48-\mathrm{C} 49-\mathrm{C} 50 \\ & \end{array}$

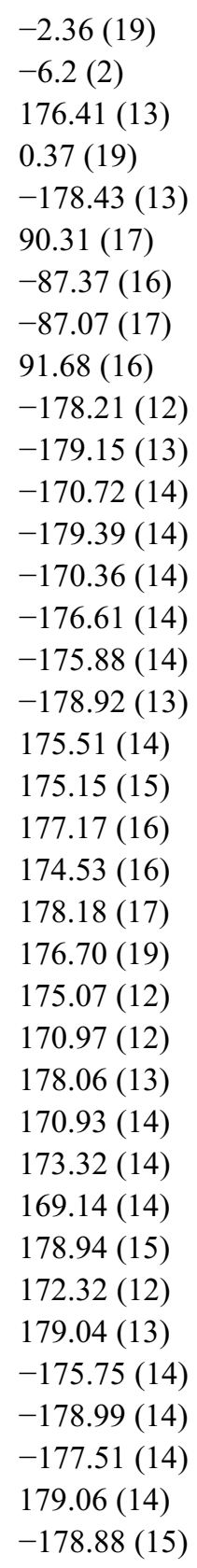

Symmetry code: (i) $-x+1, y,-z+1 / 2$.

Hydrogen-bond geometry $\left(A,{ }^{\circ}\right)$

\begin{tabular}{lllll}
\hline$D-\mathrm{H} \cdots A$ & $D-\mathrm{H}$ & $\mathrm{H} \cdots A$ & $D \cdots A$ & $D-\mathrm{H} \cdots A$ \\
\hline $\mathrm{O} 5-\mathrm{H} 5 \cdots \mathrm{O} 1$ & $0.85(2)$ & $1.93(2)$ & $2.754(2)$ & $165(2)$ \\
$\mathrm{C} 344^{\mathrm{ii}}-\mathrm{H} 34 B^{\mathrm{ii} \cdots \mathrm{Cl} 1}$ & 0.98 & 2.90 & $3.782(3)$ & 151
\end{tabular}

Symmetry code: (ii) $-x+3 / 2,-y+3 / 2,-z$. 